\title{
Aspekte einer Geschichte der deutschen Naturwissenschaftssprache und ihrer Wechselbeziehung zur Gemeinsprache
}

[1984/1986]

\subsection{Forschungsstand und Quellen}

Unsere Geschichte ist seit dem späten Mittelalter zunehmend von den Naturwissenschaften bestimmt, die naturwissenschaftlichen Fachsprachen sind daher ein wesentliches Moment neuzeitlicher Sprachentwicklung. Die Geschichte der Naturwissenschaftssprache und insbesondere die ihres Einflusses auf die allgemeine Gebrauchssprache ist aber wenig erschlossen; die allgemeinen Sprachgeschichten nehmen nur am Rand von ihr Notiz.

Eine der Ursachen liegt in der seit Beginn einer deutschen Sprachgeschichtsschreibung datierenden Kluft zwischen Natur- und Geisteswissenschaften. In den Sprachgeschichten und in der älteren Lexikographie haben die Sprache der Poesie, alter Berufsstände und versinkender Techniken und der mundartliche Wortschatz traditionellerweise einen sehr hohen Stellenwert. Die Begriffe der Naturwissenschaften sind schon deshalb oft ausgeschlossen, weil sie nicht dem ,Erbwortschatz‘ angehören. Die sog. ,Fremdwörter' sind bis weit ins 20. Jahrhundert hinein kein selbstverständlicher Bestandteil eines deutschen Wörterbuchs.

Es gibt auch Gründe, die in der Schwierigkeit der Sache liegen. Das naturwissenschaftliche Schrifttum und die von ihm infiltrierten Texte sind unüberschaubar, und wer eine Geschichte der Naturwissenschaftssprache auf diesen Quellen aufbauen will, muss von den verschiedensten Fachgebieten etwas verstehen.

Es gibt andererseits eine Fülle sprachwissenschaftlicher und fachlicher Einzelveröffentlichungen zur Sprache der Naturwissenschaften. Im Bereich der lange vernachlässigten mittelalterlichen und frühneuzeitlichen Sachprosa haben Gerhard Eis und seine Schüler zahlreiche Quellen erschlossen. Für das Verständnis der Auseinandersetzung zwischen Latein und Volkssprache ist Leonardo Olschkis Darstellung der italienischen Verhältnisse aufschlussreich. Es gibt, nicht selten von der Hand von Fachgelehrten, Monographien, z. B. zur Sprache der Mathematik (Schirmer; Busch), Physik (Gerlach), Chemie (Crosland; Wolff), der älteren Anatomie (Hyrtl) oder der Alchemie und Medizin des Paracelsus (Weimann) usw. Die fachinternen Zwecken dienenden Veröffentlichungen zum Vokabular eines Fachs sind ebenso wichtige Auskunftsmittel. Die Erforschung der Fachsprachen hat im vergangenen Jahrzehnt sehr zugenommen. Die Bibliographie von Erhard 
Barth und die Überblicksdarstellungen von Drozd \& Seibicke (mit einem instruktiven Kapitel zur ,Geschichte der Fach- und Wissenschaftssprache im Deutschen'), Fluck und Lothar Hoffmann ermöglichen einen ersten raschen Zugang.

Ein Darstellungsversuch kann außer von der genannten Sekundärliteratur von folgenden Quellen ausgehen:

a) Von Schriften wirksamer, auch öffentlichkeitswirksamer naturwissenschaftlicher Schriftsteller (wie Paracelsus, Kepler, Newton, Linné, Darwin usw.).

b) Von Überlegungen der Naturwissenschaftler zu sprachlichen Problemen, zur Form der Darstellung. Diese Äußerungen metasprachlichen Inhalts sind eine lohnende Quellengruppe.

c) Von Darstellungen der Geschichte der Naturwissenschaft in Monographien und Lexika. Diese Geschichten der Naturwissenschaft vereinfachen die Arbeit in zweifacher Hinsicht: Sie vermitteln einen Überblick über entscheidende Entwicklungsschübe in der Naturwissenschaft und erleichtern dadurch die Quellenauswahl, und sie machen recht häufig auf sprachliche Begleiterscheinungen des Wandels der Wissenschaft aufmerksam.

d) Von der Populärwissenschaft und von Wörterbüchern. - Eine Darstellung der Beziehung zwischen Sprachgeschichte und Entwicklung der Naturwissenschaften kann so vorgehen, dass sie von der allgemeinen Sprachgeschichte aus notwendig punktuell und diffus - die Entlehnungen aus den Naturwissenschaften registriert oder dass sie von den Grundlinien einer Geschichte der Naturwissenschaftssprache ausgeht und von hier aus die Übergangsstellen in die Gemeinsprache sichtbar macht. Der zweite Weg wird hier eingeschlagen. Die Sprachen der Mathematik, Physik, Chemie, Biologie werden speziell berücksichtigt.

\subsection{Faktoren der Entwicklung und Kennzeichen naturwissenschaftlicher Fachsprachen}

Die Entwicklung der Naturwissenschaften unterliegt Ausnahmebedingungen; die ,Versuchsanordnung، ist hier etwas übersichtlicher als in der allgemeinen Sprachgeschichte.

1.1 Ein wirksamer Faktor ist die Organisation der naturwissenschaftlichen Lehre an den Universitäten. Mit der Institutionalisierung eines Fachs beginnt eine eigengesetzliche Konservierung und Entfaltung des Wissens. In der bis ins 18. Jahrhundert bestehenden Vierfakultäten-Universität ist Mathematik und ,Naturkunde‘ in einem, durch einen überlieferten Autoritätenkanon umrissenen Sinn, Bestandteil des Propädeutikums der ,septem artes', (insbesondere des mathematischen ,Qua- 
driviums‘ mit seinen Fächern Arithmetik, Geometrie, Astronomie und Musik), der Philosophie und der Theologie. Theologie als ,Königin der Wissenschaften“ definiert bis in die frühe Neuzeit den Auslegungshorizont der Natur. Die sich verselbständigende Naturwissenschaft wird, soweit überhaupt, zunächst vorzugsweise im Rahmen der medizinischen Fakultät versehen; seit Ende des 17. Jahrhunderts beginnen sich die beschreibenden Naturwissenschaften, Chemie, Mineralogie, Botanik, Zoologie von der Medizin zu emanzipieren. Auch Physik wird gelegentlich eigenes Lehrfach. Erst im 19. Jahrhundert, im Zuge der ineinander wirkenden industriellen Revolution und der wissenschaftlichen revolutionären Entdeckungen, bilden die Naturwissenschaften in den Universitäten eine eigene fünfte Fakultät und beginnen, intern und öffentlich, einen Siegeszug, der sich im 20. Jahrhundert, infolge neuer revolutionärer Entdeckungsschübe und in Verbindung mit einer zweiten industriellen Revolution, fortsetzt und dazu führt, dass sie sich breitgefächert und kapitalintensiv institutionalisieren. Die einzelnen Disziplinen greifen einerseits, im mikroskopischen Bereich, verstärkt ineinander und haben sich andererseits differenziert in zahlreiche Teildisziplinen.

1.2 Entscheidende Impulse empfing die neuzeitliche Naturwissenschaft von außerhalb der Mauern der Universität. Das beginnt, was neuerdings betont wird, bei den ,artes mechanicae' und den ,artes magicae' des Mittelalters und setzt sich fort in den Anstößen, die in der frühen Neuzeit von den Bedürfnissen der Lebenspraxis, Handwerk und Technik herkommen und die vom Staat ausgehen, der aus Interesse an praktischer Nutzung u.a. naturwissenschaftliche Gesellschaften und Akademien fördert. Seit dem 19. Jahrhundert, in dem die Naturwissenschaften paradoxerweise das Prinzip reiner Welterkenntnis hervorheben, geht der stärkste Sog auf ihre Entwicklung von den Anwendungsbereichen, Wirtschaft und Technik, aus. Die Formen der Forschung, wirtschaftlich oder staatlich gefördert, haben sich zunehmend denen der arbeitsteiligen industriellen Großproduktion angeglichen.

1.3 Das Internationale erscheint weiterhin als konstanter Faktor in der Geschichte der Wissenschaftssprache; dieser Faktor ist allerdings in unterschiedlichem Grad wirksam. Zunächst, bis ins 17. und 18. Jahrhundert, war das Lateinische als europäische Gelehrtensprache Ausdruck dieser Idee. Auch nach seiner zunehmenden Ablösung durch die volkssprachige Wissenschaft blieb dieses Prinzip insofern erhalten, als ein beträchtlicher Teil des Fachvokabulars aus dem Material der alten Gelehrtensprachen, des Griechischen und Lateinischen, entnommen wurde, und es kehrt im 20. Jahrhundert wieder in der Dominanz des Englisch-Amerikanischen als Wissenschaftssprache der westlichen Welt und in den Bemühungen um ,internationale Terminologienormung، (Fluck 1976: 127ff.). - Auch die Konstruktspra- 
chen der mathematisch exakten Naturwissenschaften sind internationale Sprachen.

1.4 Schriftliche Überlieferung und Autorität der Überlieferung ist ebenfalls ein konstanter Faktor in der Geschichte der Naturwissenschaftssprache. Die Autorität kann sich an einzelne Autoren (Aristoteles z. B.) heften und als Hierarchisierung und Selektion der Überlieferung in Erscheinung treten. Sie schlägt sich nieder als Dauer und Trägheit der einmal eingeführten Sprache, was sich besonders an der älteren lateinisch formulierten Naturkunde und ihrem Autoritätenkanon beobachten lässt, aber nicht nur hier. Die sprachliche Fixierung trägt in jedem Fall zur Institutionalisierung wissenschaftlicher Vorstellungen bei und hat $\mathrm{u}$. U. die Trägheit der Institution. Wörter können als ,Idole‘ oder ,falsche Begriffe‘ (Francis Bacon) die ihnen widersprechenden Befunde überleben, das ,Phlogiston` in der Chemie (18. Jh.) und der ,Äther‘ in der Physik wären Beispiele. Das Gleiche gilt für die Sprache ganzer wissenschaftlicher Schulen.

1.5 Ein fundamentales Prinzip der Naturwissenschaftsgeschichte ist schließlich die schubweise oder allmähliche Entdeckung neuer Zusammenhänge und Gegenstände. Die wissenschaftliche Mitteilung dient nur teilweise der Verständigung über Bekanntes und mindestens ebenso sehr der Korrektur des Bekannten und der Verständigung über Unbekanntes. Das bedingt, schubweise oder allmählich, die Schaffung neuer Termini und die Verständigung über alte, die Umdeutung, Ersetzung, Erweiterung des vorhandenen Fachvokabulars. Die Geschichte der Sprache über Natur ist charakterisiert durch das Verschwinden ungezählter Wörter, der Alchemie z.B. und Astrologie, dem Bedeutungswandel naturwissenschaftlicher Begriffe (vgl. die Geschichte der Wörter ,Atom“ und „Äther‘ seit der Antike) und durch Erweiterung. Seit dem 18. Jahrhundert hat der Erkenntnisfortschritt explosionsartige Formen angenommen, und entsprechend vollzog sich die Sprachentwicklung mit sonst ungewohnter Geschwindigkeit.

1.6 Die Schaffung des Fachvokabulars ist, in der Neuzeit zunehmend, ein bewusster Akt. Er erfolgt ausdrücklich, durch die Form der Benennung (,wir nennen das“, ,wir schlagen den Namen vor') oder der Definition (,wir sprechen von Bewegung', ,Bewegung ist‘). Die Namengebung unterliegt Regeln, die einmal festgelegt worden sind und deren Befolgung überwacht wird (z.B. in der Botanik, Chemie, Ornithologie). Auch die Durchsetzung eines neuen erklärenden Begriffs unterliegt Regeln der Überprüfung auf seine Zweckmäßigkeit und Brauchbarkeit. Insofern vollzieht sich die Geschichte der Naturwissenschaftssprache - der Idee nach und auch teilweise in der Realität - nach künstlich festgelegten vernünftigen Spielregeln. Das primäre Ziel, über international vereinbarte Nomenklaturen und über 
kontextautonome, eindeutige, konnotatfreie Begriffe $\mathrm{zu}$ verfügen, setzt sich in den mathematisch exakten Naturwissenschaften fort mit der Tendenz zur Formalisierung und zu Konstruktsprachen. In dem Operieren mit Zahlen, Symbolen und Formeln, graphischen Darstellungen und Modellen ist, insbesondere in der modernen Physik (vgl. 6.1), die Grenze zur Nichtsprachlichkeit oft überschritten.

1.7 Eine Abgrenzung der historischen naturwissenschaftlichen Fachsprachen von der Gemeinsprache ist nur partiell, auf der Ebene des Fachvokabulars (bzw. Symbolinventars) und des Stils, möglich. Naturwissenschaftliche Fachsprachen sind sachbereichbezogene Subsysteme der Gemeinsprache. Man beschreibt sie am besten als Funktionalstile. Monofunktionell, dienen sie der eindeutigen und abkürzenden Verständigung über einen Gegenstandsbereich, und zwar i.e. der differenzierenden einnamigen Bezeichnung seiner Gegenstände (Nomenklaturen), seiner systematischen Ordnung durch klassifizierende Begriffe und der Erhellung der in ihm geltenden Sachzusammenhänge durch erklärende Begriffe und Formeln. Die Grenze zwischen naturwissenschaftlicher Fachsprache und Gemeinsprache verläuft, historisch wie systematisch betrachtet, auf einer gleitenden Skala. Die wissenschaftliche Terminologie bewegt sich zwischen den Polen des Zeichenterminus auf der einen und des orientierenden Terminus auf der anderen Seite. Um z.B. in einer Fachwissenschaft den Begriff ,Widerstand` zu bezeichnen, lässt sich eine Reihe von Möglichkeiten aufstellen, die beim Zeichenterminus beginnt und sich zum selbsterklärenden Ausdruck hinbewegt:

1. Frei vereinbarte Symbole $(R)$.

2. Abkürzungssymbole $(\Omega, W)$.

3. Eigennamen (Ohm).

4. Abkürzungswörter (WID, ANT).

5. Neue Lehnwörter, die aus dem lexikalischen Material der klassischen Gelehrtensprachen entnommen bzw. geprägt sind (Antistat).

6. Neue Lehnwörter, die aus einer lebenden Fremdsprache entnommen bzw. geprägt sind (engl. resistance).

7. Teilweise oder ganz eingebürgerte Lehnwörter in spezialsprachlicher Verwendung (Resistenz, Opposition).

8. Neugebildete Komposita oder feste Lexemsequenzen aus eingebürgerten Lehnwörtern (Antitendenz, resistierende Dynamis).

9. Wörter des Erbwortschatzes in spezialsprachlicher Verwendung (Widerstand).

10. Neugebildete Komposita oder feste Lexemsequenzen aus dem Erbwortschatz (Aufhaltekraft, abwehrende Kraft).

11. Metaphern (Sperre).

12. Vermeidung eines speziellen Terminus durch Synonymik, Paraphrasen und ausgeführte Beschreibungen. 
Je weiter wir in dieser Skala nach oben gehen, um so ,künstlicher' und weniger allgemeinverständlich sind die Ausdrücke, um so weniger ist ihr Verständnis aber auch beeinflussbar durch eine bereits assoziierte Bedeutung und durch die Umgebung eines benachbarten Laut- und Bedeutungsfeldes. Die Skala bezeichnet eine graduelle Entfernung der Termini von der Gemeinsprache in Richtung auf eine Spezial- und Kunstsprache und eine graduell zunehmende Terminologisierbarkeit. In den zuerst genannten Beispielen, $R, \Omega$, Ohm, ist der Ausdruck ganz oder fast ganz beliebig, ein Etikett, das frei ist für die Zuordnung vereinbarter Inhalte und durch eine Definition eingeführt und erlernt werden muss.

Je mehr man in der Skala nach unten geht, um so mehr sind die neuen wissenschaftlich intendierten Inhalte erschlossen mit Hilfe eines bekannten Inventars von Ausdrücken und ihnen zugeordneten Vorstellungen, um so mehr sind sie für den Teilhaber der Gemeinsprache motiviert und daher durchsichtig. Die Ausdrücke der Reihe von 4-11 definieren nicht, enthalten aber einen Hinweis auf den gemeinten Inhalt. In dem unter 12 genannten Beispiel der Umschreibung begrifflicher Inhalte ist die Differenz zur Gemeinsprache aufgehoben.

Je nachdem, ob sie den Zeichenterminus oder den aus bekanntem Wortgut entnommenen orientierenden Terminus bevorzugt, nähert sich die wissenschaftliche Darstellung mehr dem spezialsprachlichen Typus und der Verabredungssprache oder der Gemeinsprache. - Man kann, aufgrund dieser Skala, den Terminologietypus einer Fachsprache charakterisieren. Terminologien, die der Gemeinsprache nahestehen, haben eine erhöhte Chance, wieder in sie überzugehen, aber auch der verzerrenden Rezeption und Wirkung.

Das Verhältnis zwischen Fachstil und Gemeinsprache lässt sich analog, historisch und systematisch, als gleitende Skala beschreiben. Ein Fachgebiet hat seinen Gegenstandsbereich, seine Argumentationsformen (z.B. das Experiment und dessen Auswertung) und seine Darstellungskonventionen (z. B. die graphische Figur und die Einteilung in Paragraphen). In der wissenschaftlichen Darstellung dominiert traditionellerweise die Sachorientierung vor der Empfängerorientierung. Die Sachbezogenheit äußert sich im Vermeiden von Redundanzen, in logischer Klarheit und Exaktheit der Unterscheidungen, Ökonomie. Dem entspricht, über das fachspezifische Vokabular hinaus, eine charakteristische Auswahl, Gebrauchsfrequenz und Verwendung des allgemeinen Wortschatzes und der grammatischen (morphologischen, syntaktischen) Mittel. - Die wissenschaftliche Darstellung bewegt sich zwischen den Polen einer hohen Frequenz des Spezialwortschatzes, der denotativen, monosemantischen Terminologie und der Formalisierung, des hohen Abstraktionsgrades, der hohen Informationsdichte und der übersichtlichen Anordnung der Information auf der einen Seite und der geringen Frequenz des Spezialwortschatzes, der konnotativen und poly- 
semantischen Wortsprache, des geringen Abstraktionsgrades, der hohen Redundanz und verstreuten Information auf der anderen Seite.

Wollte man das modifizierte Sprachfunktionen-Modell als heuristisches und ordnendes Instrument verwenden, so könnte man sagen: der Idealtyp sachbezogener naturwissenschaftlicher Darstellung beruht auf strenger Selektion. In ihm dominieren die Funktionen der sachlichen Darstellung und Unterscheidung (1), der (überwiegend systematischen, deduktiv verfahrenden) Gliederung des Dargebotenen (2), der fragenden oder hypothetischen Erweiterung des gegebenen Horizonts (3), der Verständigung über den Sprachgebrauch (Metasprache) (4), während die Kontaktfunktion (5) und die poetische Funktion (6) zurückzutreten scheinen und sprachliches Ausdrucksverhalten (7) und Appellverhalten (8) möglichst vermieden werden. - Je mehr die Empfängerorientierung dominiert, um so mehr gewinnen die letzten vier - Kontakt, Ausdruck und Appell, ,poetische‘ Form - an Bedeutung und werden die ersten vier kommunikativ gehandhabt. Der Übergang aus der Fachsprache in die Gemeinsprache vollzieht sich innerhalb des zweiten Sprachtyps.

Man kann eingebürgerte Texttypen als Stufen des Übergangs zur Gemeinsprache unterscheiden. Fleck (1980: 148f.) unterscheidet z.B. drei Stufen: die esoterische Zeitschrift- und Handbuchwissenschaft, die einweihende Lehrbuchwissenschaft und die den größten Teil der Wissensgebiete eines jeden Menschen versorgende populäre Wissenschaft. Walther von Hahn (1973: 283f.) hebt die Theoriesprache (oder Wissenschaftssprache) als die strengste und vorwiegend schriftliche Form einer Fachsprache ab von der fachlichen Umgangssprache, die der direkten Kommunikation der Fachleute bei der Arbeit dient und meist gruppen- und sondersprachliche Züge annimmt, und von der Verteilersprache, die vor allem im technisch-industriellen Bereich das Fachvokabular an den Verbraucher gelangen lässt. Sie ist auch für das naturwissenschaftliche Fachvokabular eine wesentliche Vermittlungsinstanz. Habermas (1978: 328ff.) unterscheidet mit anderen die hochgradig normierte Wissenschaftssprache von den in anderen Berufsbereichen eingeführten Fachsprachen und stellt beiden die Umgangssprache der je einzelnen Sprachbenutzer und die öffentliche Bildungssprache gegenüber. Bildungssprache, die sich von der Umgangssprache durch die Disziplin des schriftlichen Ausdrucks und einen differenzierten, Fachliches einbeziehenden Wortschatz unterscheidet, dient dazu, einer Allgemeinheit Orientierungswissen $\mathrm{zu}$ vermitteln und das Fachwissen „in die einheitsstiftenden Alltagsdeutungen einzubringen“. - Populärwissenschaft, Verteilersprache, Bildungssprache sind die wesentlichen Mittler, durch die Elemente der Wissenschaftssprache in die allgemeine Gebrauchssprache hinübergehen. 


\subsection{Gelehrtenlatein, Mischprosa und frühe deutsche Sachprosa. Übergang vom Gelehrtenlatein zur deutschen Naturwissenschaftssprache}

2.1 Ein Oxforder Student des 13. Jahrhunderts studierte im Rahmen seines Grundstudiums der ,artes liberales' laut Lehrplan die Physica, Meteorologica, De caelo, De motu animalium von Aristoteles und andere Werke der bis ins 18. Jh. sog. „Naturphilosophie“. Die freien Künste waren allerdings noch überwiegend Instrumente der Theologie; die in einem fixierten Autoritätenkanon vermittelte Naturkunde diente der Erkenntnis Gottes im Sinne des ,omnis natura Deum loquitur‘ (Hugo von St. Victor). Die dem Mittelalter eigene Naturanschauung und kontemplativ-allegorische Auslegung der Dingwelt und ihre bis ins 18. Jahrhundert reichende Tradition ist in der Germanistik von Friedrich Ohly und seiner Schule erschlossen worden. Die wirksamste Naturkunde dieser Art war der ursprünglich griechische, im 11. und 12. Jahrhundert deutsch bearbeitete Physiologus. In dem neuzeitlichen Konflikt zwischen der an die Bibel und den Überlieferungskanon gebundenen Auffassung der Natur als Metapher und der Horizonterweiterung der neuzeitlichen Erfahrungswissenschaft entwickeln sich Argumentationsformen kritischer Reflexion, die geistes-, stil- und sprachgeschichtlich aufschlussreich sind (vgl. Meier 1978).

Die theologisch gebundene Naturkunde löst sich im Übergang zur systematisch sammelnden und rubrizierenden, experimentierenden und erklärenden $\mathrm{Na}$ turwissenschaft von ihrem ,philologischen' Charakter und emanzipiert sich von im Trivium vermittelten ,freien Künsten“ der Poesie und Rhetorik, deren Gewicht im Humanismus zunächst zugenommen hatte. Das zeigt sich z. B. im Stilideal der Londoner Royal Society, „alle Umschreibungen, Abschweifungen und Schwülstigkeiten des Stils zu verbannen und zu jener ursprünglichen Reinheit und Kürze zurückzukehren, mit der die Menschen so viele Dinge mit einer fast gleichen Anzahl von Worten ausdrücken konnten. Sie verpflichtete ihre Mitglieder auf einen präzisen, nüchternen, ungezwungenen Stil, auf konkrete Ausdrücke, klare Bedeutungen und eine natürliche Leichtigkeit, die alles so weit wie möglich der mathematischen Klarheit annähert“ (Heidelberger \& Thiessen 1981: 243).

2.2 Eine deutschsprachige Fachprosa beginnt schon mit den ahd. Glossen und den Übersetzungen Notkers (um 1000 n.Chr.). In Notkers interpretierenden Übersetzungen sind wie bei zahlreichen Nachfolgern die lateinischen oder griechischen Ausdrücke oft beibehalten - eine Vorform der neuzeitlichen Wissen- 
schaftssprache: „Quarum smaragdus una: déro ist éiner smaragdus, állero stéino grûonesto, fóne amaritudine geheizener, unánda îo grûone éiver [herb] ist. “ Das von Notker hier angewandte für das ganze Mittelalter charakteristische etymologische Erklärungsverfahren ist in anderer Form, als Verfahren laienhaften wörtlichen Verstehens wissenschaftlicher Termini, bis in die Gegenwart von kaum zu überschätzender Bedeutung. Seit dem späten Mittelalter (13. Jh.) und nach dem Buchdruck existiert, wie die Pionierarbeit von Gerhard Eis und u.a. die Arbeiten seiner Schüler Gundolf Keil und Peter Assion zeigen, eine umfangreiche und dicht verbreitete Sachprosa, die in den Lexika bisher kaum gebucht ist und deren vollständige Edition und Erschließung das bisherige Bild von der Entstehung der neuhochdeutschen Schriftsprache vermutlich korrigieren wird. Hierher gehören, aus der Reihe der sieben ,artes liberales' z.B. Schriften wie Konrad von Megenbergs (1309-1377) Buch der Natur oder die Geometria deutsch, aus dem Bereich der weit ergiebigeren sieben ,artes mechanicae“ das 1. Fach ,Handwerk', mit Schriften für Färber z.B., zur Alchemie, Bergbüchlein, das 5. Fach ,Wald und Tiere' mit Jagdliteratur, Büchlein zur Rossheilkunde z.B. oder zum Angeln und Fischen, das 6. Fach ,Heilkunde' mit Arzneibüchern, Kräuterbüchern, Steinbüchern, Syphilistraktaten u. a.m. Die Schriften sind großenteils Übersetzungen aus dem Lateinischen, auch noch im 16./17. Jahrhundert, wo der Strom derartiger ,Kunstbücher` sich verbreitert. Die historische Grundform war das Rezept, der Anweisungsstil der oft an Sprechsprache gebundenen Ratgeber: „Swen die binen oder di wespen stechen, der sal uf di stat legen, da si in gestochen haben, alcativam unde girstin mel getempert mit ezzige.“ Es gibt stereotype sprachliche Formeln, die jahrhundertelang überliefert werden. Neben den Prosagattungen, vgl. auch den Traktat oder den ,Schreizettel' (Reklameblatt), reicht das naturkundliche Lehrgedicht bis ins 18. Jahrhundert (Goethe).

Der Wortschatz ist reich. Seine Quellen sind neben den klassischen Gelehrtensprachen auch das Arabische, aus dem z.B. das griechische oder ägyptische ,chemeia', (arab. ,al-kimiya') als ,Alchemie‘ zurückkehrte. Die alchemistische Fachsprache (Arkansprache) war absichtlich dunkel, um Unbefugte und Nichteingeweihte von dem geheimen und $\mathrm{u}$. U. gefährlichen Wissen fernzuhalten. Man gab z.B. den sieben Metallen die Namen der sieben Planeten, nannte Gold ,Sol', Kupfer ,Venus‘, Quecksilber ,Mercur' oder bezeichnete sie durch die Symbole $\odot$, \%, ๖, und stellte chemische Umwandlungen dar, indem man sie durch Symbole, Metaphern, mythologische Personifikationen verrätselte.

Eine sprachgeschichtlich wirksame Gestalt der frühen Neuzeit neben Luther war Theophrastus Bombastus von Hohenheim, genannt Paracelsus (1493-1541), der als berühmter und umstrittener Wanderarzt, bevor Christian Thomasius die deutschsprachige Vorlesung in Leipzig (1667) begann und später in Halle durchsetzte, 1527 in Basel eine deutschsprachige, d.h. deutschlateinische Vorlesung 
über die Chirurgia Vulnerum hielt, die ein Schüler aufzeichnete: „Si vis curari, noli sprützen aquam in die Fistell“.

Wie die Entwicklung der neuzeitlichen Naturwissenschaften dem praktischen Bereich, Handwerk und Technik, starke Impulse verdankte, so entsprang auch das Übersetzen praktischen Bedürfnissen. Paracelsus schrieb seine Grosze Wunderartzney 1536 für Bader und Wundärzte, meist Ungelehrte, die ihr Handwerk in einer Lehre lernten. Er gebraucht in seinem umfangreichen, verbreiteten Schrifttum, das zurzeit noch lexikographisch aufgearbeitet wird, zahlreiche lateinische Termini und bildet neue, assimiliert das fremdsprachliche Vokabular, übersetzt es und erfindet neue deutsche Wörter. Es gibt anscheinend kaum einen wissenschaftlichen Autor, der den deutschen Wortschatz derartig erweitert hat wie der im Grenzgebiet zwischen Alchemie und Heilkunst angesiedelte Paracelsus: ,Alkohol' und ,Gas‘, ,Homunculus‘, ,Kobalt‘ und ,Zink', ,Sylphe‘ und ,Undine‘, ,Aphorismen', ,alchimistisch', ,Attraktion', ,elektrisch“, ,Elixier‘, ,Infektion‘, ,metallisch“, ,Mikrokosmos‘, ,Natron‘, ,Paroxysmus‘, ,Signatur', ,Sublimierung‘, ,Tinktur‘, aber auch Neubildungen aus dem Erbwortschatz wie ,Amtsträger‘, ,Eiweiß‘, ,Erbkrankheit‘, ,Erkältung“, ,Frauenkrankheit‘, ,Geisteskrankheit‘ und ,Seelenkrankheit' haben sich möglicherweise durch ihn eingebürgert (nach Weimann 1963; anders Telle 1981).

2.3 Der Übergang von der lateinischen zur landessprachlichen Naturwissenschaft vollzog sich im deutschen Universitätsbereich im Vergleich $\mathrm{zu}$ den Nachbarländern - Italien, Frankreich, England - und zu den meisten anderen gelehrten Disziplinen spät, nämlich in der zweiten Hälfte des 18. Jahrhunderts. Er wurde von Leibniz schon 1676 (?) in einer lateinischen Denkschrift über die Förderung der angewandten Naturwissenschaften (Consultatio de naturae cognitione ad vitae usus ...) unter dem Leitgedanken einer allgemeinen Aufklärung gefordert (Leibniz 1916a: 86). In einem deutschen Aufsatz Ermahnung an die Teutsche, ihren verstand und sprache besser zu üben (1682/83?) diagnostiziert Leibniz im Anschluss an die Sprachgesellschaften des Barock eine Sprachkrise er meint die deutsch-französische ,Sprachmengerei ${ }^{\star}$ - sieht aber nicht wie die Sprachgesellschaften in der Poesie das Mittel, dem Zustand der Sprache aufzuhelfen, sondern entdeckt die Bedeutung der Sachprosa, fordert den Übergang vom Gelehrtenlatein ins Deutsche und die Ausarbeitung eines Kanons von Sachprosaschriften. In dem Aufsatz Unvorgreifliche Gedanken, betreffend die Ausübung und Verbesserung der Teutschen Sprache (1697) entwickelt er die Idee einer umfangreichen Wörterbucharbeit und projektiert in diesem Rahmen ein umfassendes, für den internationalen Vergleich bestimmtes Fachsprachenlexikon, einen ,Schatz‘ der ,Kunst-Worte“ oder ,cornu copiae‘ (§33), den er einer zu gründenden Akademie anvertrauen möchte, in der sich, wie er hofft, die bestehenden Natur- 
wissenschafts- und Sprachgesellschaften vereinigen sollen. - Von ebenso grundsätzlicher Bedeutung sind Leibniz’ Überlegungen zu lateinischen und deutschen ,Kunstausdrücken“ oder ,Technica‘ in seiner frühen Schrift De optima philosophi dictione (1670). (Der von Paracelsus 1537/38 eingeführte Ausdruck ,Terminus‘ oder ,termin' wird übrigens im 17./18. Jahrhundert neben ,Kunstausdruck', meist in der Verbindung ,technicus‘ oder ,technologicus‘, gebraucht; das Wort ,Terminologie‘ bürgert sich anfangs des 18. Jahrhunderts ein.)

Der Übergang vom lateinischen zum deutschen Schrifttum vollzog sich insgesamt in einem Zeitraum von 300 Jahren. 1518 war in Deutschland nur $10 \%$ der Buchproduktion deutsch, 1681 überragte zum ersten Mal die Zahl der deutschen die der lateinischen Publikationen, Ende des 18. Jahrhunderts waren nur noch $4 \%$ oder 5\% lateinisch (vgl. Bach 1965: 309). Aus den Untersuchungen zum deutsch-lateinischen Büchermarkt von Rudolf Jentzsch, der schon 1912 die Leipziger Ostermesskataloge als Barometer eines Epochen- und Publikumswandels entdeckte, ergibt sich für die von ihm untersuchten Stichdaten des 18. Jahrhunderts: Der Anteil der mathematischen Schriften entspricht um 1740 dem der naturwissenschaftlichen (1,5\%) und steigt bis 1800 proportional zum übrigen Schrifttum, ihre Sprache ist aber im Gegensatz zu den Naturwissenschaften schon 1740 deutsch. Deutsche Rechenbücher oder die Geometria deutsch gibt es schon im Spätmittelalter; die praktischen Bedürfnisse machten sich hier früh geltend. Die ersten starken Impulse empfing die deutsche mathematische Fachsprache aus der Malerei und Fassmesskunst. Albrecht Dürer veröffentlichte 1525 eine Underweysung der Messung, eine Art Leitfaden für Malerknaben, der sie in Fragen der Perspektive und der Konstruktion geometrischer Figuren unterrichtet. Das Werk enthält knappe Konstruktionsbeschreibungen und eine erste deutschsprachige mathematische Terminologie. An Stelle von ,conus', ,sphaera', ,cubus' sagt Dürer ,Kegel', ,Kugel' und ,Würfel'. Er verwendet kaum den an sich häufigen Typ der Lehnübersetzung. Für ,diameter sagt er z.B. nicht ,Durchmesser', sondern ,Zwerchlinie‘. Er bemüht sich um Neubildungen und zielt dabei auf Anschauung: „Die Elipsis will ich eine Eierlinie nennen, darum daß sie schier einem Ei gleich ist. Die Parabola sei genannt eine Brennlinie, darum so man aus ihr einen Spiegel macht, so zündet sie an. Aber die Hyperbola will ich eine Gabellinie nennen“ (zit. nach Olschki 1919: 441).

Der Ansatz Dürers ist von Johannes Kepler systematischer ausgearbeitet worden, in einer deutschen Fassung seiner Nova Stereometria doliorum von 1615, einer Schrift, die dazu anleitete, den Rauminhalt von Fässern zu bestimmen. Kepler veröffentlichte diese seine Fassmesskunst (Messekunst Archimedis) 1616 auf deutsch, um die deutschen Behörden, Weinvisierer und Küfer anzusprechen. Er tritt aber auch grundsätzlich für eine deutsche mathematische Terminologie ein. Aus diesem Weinvisierbuch sind zahlreiche Termini in die mathematische 
Fachsprache eingegangen: terminologisierte Ausdrücke der Umgangssprache, ,Länge‘, ,Breite‘, ,Höhe‘, ,Gleichheit‘, aber auch Neubildungen: ,Beweis‘, ,Gleichheit‘, ,ähnlich‘, ,kugelrund‘, ,Kegelschnitt‘, ,Querschnitt‘, ,Spirallinie‘. Es wäre aber auch aufschlussreich, der Frage nachzugehen, welche Ausdrücke nicht angenommen wurden und warum nicht: Kepler sagt für ,Ellips' ,ablenger Circkel ${ }^{\text {‘ }}$ oder ,Ovallinie‘, für ,Segment‘ ,Schnitz‘, für ,Parallele‘,Gleichlaufende‘ (vgl. Götze 1919).

Die Schriften Dürers und Keplers hatten Erfolg in ihrer lateinischen Fassung, nicht in der deutschen. Eine deutsche mathematische Fachsprache hatte erst eine Chance, sich durchzusetzen, als man im 18. Jahrhundert auch an Universitäten zu einer deutschen Wissenschaftssprache übergegangen war. Christian Wolff, der wie Thomasius in Halle lebte, wurde ihr eigentlicher Begründer. Er führte eigene Termini ein, ordnete und definierte jedoch hauptsächlich das vorhandene Vokabular. Wolff war kein Purist. Er verwendete assimilierte Fremdwörter wie ,Quotient', ,Quadrat' neben deutschen Wörtern wie ,Zahl‘, ,Bruch', ,Nenner', schuf einige Neubildungen wie ,Nebenwinkel‘, ,Brennpunkt‘, ,Spielraum‘, ,Versuch', ,Schwerpunkt‘, ,Hebel', ,Abstand', ,Geschwindigkeit', und gebrauchte den einmal gewählten Terminus definitiv und konstant. Seine Anfangsgründe aller mathematischen Wissenschaften (1710) und sein Mathematisches Lexikon (1734) waren grundlegend und wurden durch J.H. Lambert um die Mitte des 18. Jahrhunderts noch einmal durchgearbeitet und ausgebaut (vgl. Busch 1933).

Kaum weniger wirksam war er durch seine neue wissenschaftliche Prosa, in der er sich von Poesie und Rhetorik so weit wie möglich trennt. Er definiert Begriffe, um mit ihnen als fest umrissenen Bausteinen $\mathrm{zu}$ arbeiten, schreibt emotionslos, systematisch und in der erklärten Absicht, die Begriffe kontextunabhängig, konstant und konnotatfrei zu verwenden, ihren latenten Bildgehalt auszublenden. Nichts darf der Phantasie überlassen bleiben, Wiederholung stört nicht, auch nicht die rhythmische Monotonie der einfachen Parataxe. Das Resultat ist, erstmals in deutscher Sprache, ein von überschaubaren Spielregeln geleiteter, klarer, methodischer Stil.

Wolff ist zugleich ein Beispiel, dass die Durchsetzung einer Wissenschaftssprache abhängig ist von ihrer Eignung zur Schulbildung und von tatsächlicher Schulbildung. Seine entschiedene, definitive Sprache und Wirkung gehören offenbar zusammen; außerdem übt Wolff Macht aus durch seine zahlreichen Schüler auf den Lehrstühlen des 18. Jahrhunderts.

In den Naturwissenschaften - Physik, Chemie, Mineralogie, Botanik, Zoologie - vollzieht sich demgegenüber der Übergang zum Deutschen erst spät im 18. Jahrhundert. Ihr Anteil am gesamten Messeangebot ist 1740 verschwindend gering (12 von 777 Schriften, 1,5\%) und ihre Sprache noch zu zwei Drittel lateinisch (8 : 4). 1770 ist ihr Anteil um das Dreieinhalbfache gestiegen (44 von 1144 
Schriften, $4 \%$ ) und ihre Sprache nur noch zu einem Drittel lateinisch (14:28). 1800 hat sich der Anteil der naturwissenschaftlichen Schriften noch einmal verdreifacht (129 von 2569 Schriften, 5\%), die lateinischen haben sich auf ein Sechstel reduziert (21:108). Während der Markt um 1740 noch ganz überwiegend fachgelehrt ist, lassen sich 1800 „die Grenzen zwischen wissenschaftlich-gelehrten und populären Schriften äußerst schwer, oft überhaupt nicht ziehen“ (Jentzsch 1912: 320). In dieser Epoche werden die lateinisch schreibenden Schweden Linné und Bergman, die Franzosen Bonnet und Buffon ins Deutsche übertragen und Adel und Bürgertum werden, wie wir aus Weimar genau wissen, zu einem naturwissenschaftlichen Publikum. Ist der verspätete Übergang eine wesentliche Ursache für die traditionelle Sonderstellung der sog. ,Fremdwörter im deutschen Sprachbereich, für die Sprachenkluft zwischen Gelehrten und Laien, für die lange Lehnprägung der deutschen Gelehrtensprache durch die lateinische? - Wir haben in Leonardo Olschkis dreibändiger Geschichte der neusprachlichen wissenschaftlichen Literatur ein überragendes, geistreiches Beispiel für die Darstellung eines solchen Prozesses im italienischen Sprachgebiet zwischen Dante und Galilei.

Der Übergang vom Gelehrtenlatein zum Deutschen, die Demokratisierung der Wissenschaft war ein Prozess von nicht zu unterschätzender Dynamik. Eine enorme Erweiterung des Wortschatzes, des Ausdruckshorizonts der Gemeinsprache, war die Folge. Die geistigen Güter konnten, durchaus im Sinn von Leibniz, zirkulieren. $\mathrm{Zu}$ den Folgen gehört auch die vielbewunderte Sachprosa des 19. Jahrhunderts. - Aber die Einheit der gelehrten Universalsprache, der ,lingua Europaea universalis et durabilis ad posteritatem', die Leibniz erhalten wissen wollte, zerbrach. Sie dauerte nur fort in den aus griechisch-lateinischem Wortmaterial gebildeten Terminologien, u. a. der Medizin, Chemie, Biologie.

\subsection{Inauguratoren neuer Fachsprachen und ihre Wirkung. Linné, Newton, Lavoisier und die Terminologietypen der Biologie, Physik, Chemie}

Man kann beobachten, dass die Begründer einer wissenschaftlichen Richtung relativ allgemeinverständlich schreiben - am Beginn der biologischen Evolutionslehre, der Psychoanalyse oder der Verhaltensforschung stehen weitgehend gemeinsprachliche Darstellungen. Das erklärt sich zum Teil daraus, dass Autoren wie Darwin, Freud, Lorenz sich noch nicht an ein Fachpublikum im engeren 
Sinne wenden können; sie müssen die Einsicht in neu entdeckte Zusammenhänge und eine entsprechende Terminologie erstmalig vermitteln und können in dieser Hinsicht nichts voraussetzen. Das bedingt eine Sprache, die einführt und erklärt, an das Bekannte anknüpft, von hier aus die neuen Theorien entwickelt. - Teils erklärt es sich auch daraus, dass die lnauguratoren, von der allgemeinen anthropologischen Bedeutung ihrer Entdeckungen überzeugt, sich von vornherein an ein allgemeines Publikum wenden wollen.

Ein Kennzeichen junger Fachsprachen, darauf hat Helmut Gipper (1969: 70ff.) aufmerksam gemacht, ist speziell ihr Reichtum an Metaphern: die Geschichte der Anatomie beginnt mit grober Metaphorik und anthropomorphen Vorstellungen, Faraday und Einstein seien zwei illustre Beispiele dafür, wie mit Hilfe anschaulicher Bilder und Metaphern neue Sehweisen versprachlicht werden könnten. Auch hier leuchtet der Zusammenhang ein. Was zu sagen ist, ist unbekannt. Eine Aufgabe der Metapher ist aber, Unbekanntes mit Hilfe bekannter Vorstellungen zu erschließen.

Vielleicht ist für diese Gemeinsprachlichkeit auch ein besonderes Verhältnis des wissenschaftlichen Erneuerers zur Sprache verantwortlich. Die Begründer einer neuen wissenschaftlichen Richtung haben ein in gewissem Sinn unabhängiges Verhältnis zur überlieferten Sprache. Dass sie sich ihre Begriffe nicht von der überlieferten Sprache vorschreiben lassen, (auch nicht von ihrer eigenen), sondern sich den Sachen neu gegenüberstellen, sie von der Gemeinsprache her neu durchdenken, ist eine Vorbedingung ihrer neuen Begriffe. Das sozusagen sprachunabhängige, unmittelbare Verhältnis zur Sache ist sehr auffallend.

Dem entspricht auf der anderen Seite das aktive, umgestaltende Handhaben der Sprache: die Neuprägung von Begriffen, die Schaffung von Termini und Nomenklaturen. Darin wiederum liegt eine Entfernung von der Gemeinsprache. Der Autor ist Gesetzgeber, der die Spielmarken, mit deren Hilfe er die Gegenstände und Sachzusammenhänge in der Natur abbildet, selbst macht und erprobt und zurechtschneidet. - (Identitätsschwache Fächer haben die Neigung zu erhöhter, sozial abgrenzender Terminologiebildung.)

3.1 Linné war ein solcher Gesetzgeber, der - kaum originär - einer Überlieferung eine definitive Gestalt gab. „Wenn man Autoren vor und nach Linnés Reformation liest, findet man eine ganz verschiedene Sprache“ schreibt er über sich selbst in einer postum veröffentlichten Aufzeichnung. Er brachte Ordnung in die verwirrende Vielgestaltigkeit der Pflanzenwelt und in die uneinheitliche Sprache der Botaniker durch seine strenge Methode der Einteilung und Benennung. In seinem Werk Systema Naturae, das 1735 zum ersten Mal erschien, verwandte er erstens ein übersichtliches Klassifikationssystem, das später erweitert wurde, aber bis heute grundlegend blieb. Er teilte das Pflanzenreich ein in sukzessiv kleiner 
werdende Rubriken, in Klassen, Ordnungen, Gattungen und Arten. Die Einteilung erfolgte aufgrund fortschreitender Übereinstimmung in den Blütenteilen. Er wandte seine Begriffsleiter - Klasse, Ordnung, Gattung, Art - mit annähernd gleichem Erfolg auch auf das Tierreich an und gilt für die gesamte Biologie als Begründer der Taxonomie. Voraussetzung der logischen Klassifikation war, dass die Blütenorgane, die den Schlüssel (,Clavis`) der Einteilung bildeten, exakt unterschieden und benannt wurden. Darin bestand die zweite bedeutsame Leistung Linnés, dass er die unterscheidbaren Pflanzenteile und ihre typischen Formen durch ein detailliert aufgefächertes Wortfeld - von der ,Wurzel` bis zur „Narbe‘ - abdeckte. Drittens begründete Linné eine einheitliche Benennung der Arten. Aus den Pflanzennamen waren vor Linné, angesichts der wachsenden Zahl von bekannten Arten, zunehmend längere Beschreibungen geworden. Die Form der Benennung war oft inkonsequent und undurchsichtig; es war zu vielen Synonyma gekommen. Die rote Johannisbeere hieß bei einem Vorgänger Linnés ,grossularia, multiplici acino: seu non spinosa hortensis rubra, seu Ribes officinarium‘. Linné gab ihr den Namen ,Ribes rubrum‘. Seit ihm werden die Pflanzennamen durch einen lateinischen Doppelnamen bezeichnet, der erste bezeichnet die Gattung und der zweite, im Sinne eines Eigenschaftswortes, die besondere Art: ,Viola tricolor‘, ,Viola mirabilis‘. Es ist das klassische Verfahren der Definition, ,per genus proximum et differentiam spezificam‘.

Die von Linné in der Philosophia Botanica aufgestellten Regeln einer ,binären Nomenklatur‘ sind in den Grundzügen bis heute gültig. Sie haben ein nicht anzugebendes Ausmaß an Verwirrung, das sonst entstanden wäre, von vornherein vermieden.

Die neue präzise Kunstsprache, die Klassifikationsmethode und verbindliche Nomenklatur, ermöglichte wohl erst eine immense Ausweitung des Wissens. Sie wurde zum Suchgerät, das von zahlreichen Schülern leicht und sicher gehandhabt werden konnte. Die Erstauflage von Linnés Systema Naturae war ein großformatiges Heft von 11 Folioseiten und erfasste 549 Tierarten. Das Werk wurde ständig erweitert und neu aufgelegt. Die 10. Auflage erschien 1766/68 - sie wurde noch von ihm selbst besorgt und umfasste drei starke Bände - über die Tiere, die Pflanzen und Mineralien. Sie erfasst 5897 Tierarten und schätzt die Zahl der Pflanzen auf 10.000. Heute rechnet man mit 11/4 Millionen Tierarten, darunter mehr als 750.000 Insektenarten, und geht von 370.000 bis 380.000 Pflanzenarten aus, die wissenschaftlich abgegrenzt werden müssen und daher stehender Benennungen bedürfen.

Linné schrieb in der Regel Latein; sein Systema Naturae wurde in der zweiten Hälfte des 18. Jahrhunderts mehrfach erweitert ins Deutsche übertragen. Die Übersetzungen und Erweiterungen Linnés wären ein ergiebiges Arbeitsfeld. Die deutsche Bildungssprache ist sicher durch seinen Einfluss bereichert worden. 
Dieser Einfluss ist bei Adelung nur teilweise und in Ansätzen greifbar; in dem Wörterbuch von Campe, das $1807 \mathrm{f}$. erschien, ist er dann aber deutlich ausgeprägt. Campes Erläuterungen der Begriffe ,Klasse‘, ,Ordnung‘, ,Gattung‘ und ,Art‘ geben jeweils auch die Sonderbedeutung an, die ihre lateinischen Äquivalente ,classis‘, ,ordo‘, ,genus‘, ,species‘ bei Linné haben - und zwar in deutlicher Anlehnung an Linné. So spricht Campe z.B. in dem Artikel ,Klasse“ von der „Naturbeschreibung, wo die Naturreiche in Klassen, und namentlich das Tierreich in sechs Klassen, die Säugetiere, die Vögel, zweilebige Tiere [das ist eine Lehnübersetzung von Linnés ,Amphibia'], Fische, Ziefer [eine Eindeutschung von Linnés ,Insecta'] und Würmer eingeteilt werden. Diese Klassen werden wieder in Ordnungen, diese in Geschlechter, Gattungen und Arten abgeteilt“. Für Linnés Bezeichnung der Pflanzenteile ,Calyx‘, ,Corolla‘, ,Stamina‘, ,Filamenta‘, ,Anthera‘, ,Pistill‘, ,Stylus‘, ,Stigma‘ erscheinen in Campes Wörterbuch als ihre deutschsprachigen Äquivalente ,Kelch“, „Krone‘, ,Staubgefäß‘, ,Staubfaden“, ,Staubbeutel“, ,Fruchtknoten“, ,Griffel, ,Narbe‘, teils also neue Übersetzungen, teils Sonderbedeutungen gebräuchlicher Wörter.

Müllers Übersetzung (Linné \& Müller 1773) prägte für die 39 von Linné benannten Spinnenarten, die in der Volkssprache bis dahin kaum unterschieden wurden, deutsche Namen. Noch Lorenz Oken erfand oder verbreitete in seinem Lehrbuch der Naturgeschichte (1815) eine Fülle deutscher Namen, von denen einige wie ,Lurche‘, ,Kerfe‘, ,Echse‘, ,Schleiche‘ sich einbürgerten. Es gab also eine Eindeutschung der wissenschaftlichen Biologie. Kunstnamen wie ,Korbblütler‘, ,Lippenblütler‘, ,Kreuzblütler‘ wurden in dieser Übersetzung Bildungsgut. Aber derartige Bemühungen wurden, angesichts der rapide wachsenden Zahl bekannter Arten, (man unterscheidet heute mehr als 20.000 Spinnenarten), seit der Mitte des 19. Jahrhunderts sehr viel seltener.

Linné war eine Institution des 18. und auch noch des beginnenden 19. Jahrhunderts. Mit der Institutionalisierung einer Sprache kann die einer Sehweise verbunden sein. Goethe war der Meinung, dass Linnés definitive, statistisch klassifizierende Sprache seine Wirkung begünstigt, aber den Blick auf die Übergangshaftigkeit der Phänomene - auf das Pflanzenreich als bewegliches Kontinuum - behindert habe. Der Übergang von der älteren systematischen ,Naturgeschichte' zur evolutiven ,Geschichte der Natur', der sprachhistorisch beschreibenswerte Prozess der ,Verzeitlichung، (Lepenies 1976) im 19. Jahrhundert, setzt mit einer Kritik an den definitiven Distinktionen Linnés ein.

Seine Begriffsleiter, sein taxonomisches System wird weiter ausgearbeitet, ausdifferenziert, verändert; die einzelnen Begriffe, ,Klasse‘, ,Ordnung‘, ,Art‘, werden strenger terminologisiert. Der Vorgang wiederholt sich in der Geschichte des Faches. Ausdrücke, die zunächst noch gemeinsprachlich verwendet werden und die der Vorgänger intuitiv zu verstehen glaubte - ,Same‘, ,Nische“ - werden vom 
Nachfolger in Frage gestellt, diskutiert, neu definiert: sie erhalten einen engeren, genormten Sinn. So ergibt sich fast automatisch ein Zuwachs an Metasprache und eine Sprachdifferenzierung. (Ohnehin verhalten sich die Schüler in der Regel terminologischer als die Lehrer.)

Man könnte hier an Thomas S. Kuhns der Naturwissenschaftsgeschichte abgewonnene Vorstellung von der Struktur wissenschaftlicher Revolutionen anknüpfen: ein Paradigma, ein einmal entworfenes Denk- und Erfahrungsmodell, wird von den Nachfolgern ausgearbeitet, durch breite, ins Detail gehende Forschung eingeholt und erweitert und verbreitet. Geschichte der Naturwissenschaftssprache wäre dementsprechend Vervielfältigung, Variation und Modifikation eines einmal entworfenen sprachlichen Musters, seine Erweiterung und Ausdifferenzierung bis zur Krise. Das bedeutet unvermeidlich Spezialisierung der Fachsprachen und Entfernung von der Gemeinsprache.

3.2 Als Paradigma im Bereich der klassischen Physik lassen sich auf ähnliche Weise die Mechanik und Optik Newtons (1642-1727) beschreiben. Newtons Formulierung des Gravitationsgesetzes, nach dem die anziehende Kraft von Körpern ihrer Masse direkt und dem Quadrat ihrer Entfernungen umgekehrt proportional ist, war so grundlegend wie seine Versuchsreihe zur Zerlegung des Lichts, zu den unterschiedlichen Brechungswinkeln der Spektralfarben und ihrer Wiedervereinigung zu weißem Licht. Charakteristisch ist für die Sprache in diesem Bereich die Verallgemeinerung und abstrakte Fassung von Grundbegriffen wie ,Kraft‘, ,Masse‘, ,Schwere‘, ,Bewegung', ,Trägheit‘, ,Geschwindigkeit‘, ,Geschwindigkeitsänderung‘. In der deutschsprachigen Physik erscheinen sie als terminologisierte Alltagsbegriffe. Ebenso charakteristisch ist das zeitlose, allgemeingültige Sachverhalte bezeichnende Praesens: „Eine von außen einwirkende Kraft ist ein Einfluß, der auf einen Körper ausgeübt wird, um seinen Zustand, und zwar den der Ruhe oder den der gleichförmiggeradlinigen Vorwärtsbewegung, zu verändern“ (Newton, Principia mathematica).

Newtons lateinische Mechanik, die Philosophia naturalis principia mathematica (1687) und seine englischsprachigen Optics (1704) wurden erst im letzten Drittel des 19. Jahrhunderts ins Deutsche übersetzt, während seine theologischen Schriften schon 1765 übertragen wurden. Zentrale Begriffe seiner physikalischen Werke tauchen aber schon zu Beginn des 18. Jahrhunderts in Werken auf, die sich an eine breitere Leserschaft wenden. Zedlers Universal-Lexikon (1735) enthält z.B. über „Gravitas, die Schwere“, einen 45spaltigen Artikel, der Newton referiert und den Begriff ,vis centrifuga‘ durch ,Centrifugal-Kraft‘ wiedergibt, während er ,vis centripeta' beibehält und ,Gravitation' noch als ,die Schwerdrückung' übersetzt. Vermittelt u.a. durch französische Adaptionen, durch Werke von Clairault, Lagrange, Laplace, die in der zweiten Hälfte des 18. Jahrhunderts ins Deutsche 
übersetzt wurden, gehen die Begriffe der Mechanik Newtons in die allgemeinen Wörterbücher und in die Gebrauchssprache ein. Adelung (1798) hat ,Gravitation“= ,Schwerkraft', ,Attraktion“=,Anziehung, Anziehungskraft', Campe hat in seinem Verdeutschungswörterbuch (1801) die gleichen Eindeutschungen und gibt die Fremdwörter ,Centrifugalkraft' und ,Centripetalkraft' wieder als ,Anstrebekraft" und ,Abstrebekraft‘. - Auch Begriffe der Optik wie ,brechen‘, ,Brechung‘ sind bei Adelung und Campe erklärt. Campe übersetzt zudem in seinem Verdeutschungswörterbuch ,Reflexion“ als ,Zurückwerfung und Zurückprallen, Rückprall‘, ,Prisma‘ als ,Ecksäule, Lichtspalter', ,Refraktion“ als ,Strahlenbrechung،, ,Strahlenbruch‘. - ,Spektrum‘ (,Spektralfarben`) ist bei Adelung, Campe, Grimm nicht belegt; das englische Wort ,spectre‘ (,Gespenst', ,Phantom‘, ,Erscheinung'), das erst durch Newton seine heute gültige Bedeutung erhielt (vgl. Gerlach 1962: 43f.), erweist schon durch die von ihm entfaltete wortbildungsmäßige Produktivität, in welchem Maß das Newton'sche Modell auf Erweiterung und Vervielfältigung angelegt ist: ,Spektralanalyse‘, ,Spektrallinie‘, ,Spektralklasse‘, ,Spektrograph'; es wird in vielfachen Übertragungen im Bereich optischer und akustischer Frequenzen gebraucht. Gerlach macht auf eine vergleichbare Produktivität der Begriffe ,Strahlen', ,Quanten‘, ,Röntgen‘ aufmerksam (,Röntgenstrahlen‘, ,Röntgenröhre‘, ,Röntgeneinheit‘, ,Röntgenfilme‘, ,Röntgenschwestern‘, ,Röntgenärzte‘, ,Röntgenschirme‘, ,Röntgenverbrennung‘, ,röntgen‘, ,Röntgenreihenuntersuchungen' usw.) (vgl. Gerlach 1962: 26, 35ff., 59f.).

Die Erfindung eines Apparats, des Spiegelteleskops durch Newton oder der Röntgenröhre durch Röntgen, und die Veränderung des Naturbegriffs werden zum allgemein bekannten Sachgut bzw. Bildungsgut - dieser Vorgang wiederholt sich ständig und führt dazu, dass ein unermessliches Vokabular aus den Naturwissenschaften in den Bereich der Bildungssprache oder allgemeinen Gebrauchssprache übergeht. Die physikalische Terminologie seit Newton ist ein Mischtyp; sie enthält gemeinsprachliche Ausdrücke wie ,Masse‘, ,Kraft‘, ,Zeit‘ zur Bezeichnung physikalischer Größen, griechische und lateinische Formen wie ,lux‘, ,lumen‘, ,stilb‘ (für photometrische Einheiten) oder ,dyn' und ,erg‘ (für Kraft und Energie). Sie bildet aus Elementen dieser Sprachen neue Fachwörter wie ,Ion', ,nuclear‘, ,Positron', ,Elektron‘ oder sie benennt neue Sachverhalte und Entdeckungen nach ihren Erforschern: ,Faraday-Effekt‘, ,Volt‘, ,Ohm‘, ,Celsius‘. Auch Apparate, wie z.B. der ,van de Graaf‘ (zur Erzeugung höherer Spannung), werden nach ihrem Erfinder benannt. Physikalische Einheiten werden wiedergegeben durch Kürzung eines Eigennamens, ,Torr` für die Druckeinheit (aus Torricelli), durch die Anfangsbuchstaben des Fachworts wie ,s‘ (für die Zeitsekunde), ,cm` (für Zentimeter). Mit der Entwicklung des Englisch-Amerikanischen zur naturwissenschaftlichen Weltsprache nimmt das aus dieser Sprache entlehnte Vokabular zu: ,bubble chamber‘, ,breeder‘, ,Reaktor‘, ,Geiger-Müller-Counter‘. 
Alle diese terminologischen Möglichkeiten haben Eingang gefunden in die Gemeinsprache.

3.3 Der Übergang von der Alchemie, der noch Newton anhing, zur neuzeitlichen Chemie vollzog sich im 18. Jahrhundert. Eine der Nachwirkungen Newtons war, dass sein Begriff der ,attractio“ in die Chemie übertragen wurde. Torbern Bergmans De attractionibus electivis (1775) stellt die Trennung chemischer Verbindungen in ihre Elemente und deren Wiederverbindung mit anderen Elementen mit Hilfe des Begriffs stärkerer ,Anziehung` dar. Der Begriff ,attractio electiva` wurde seit 1779 deutsch mit ,Wahlverwandtschaft‘ oder ,Wahlanziehung“ wiedergegeben. Er war bis zum Herbst 1809 nur als Terminus in der Chemie gebräuchlich danach erschien Goethes Roman Die Wahlverwandtschaften, der dieses ,ethische Gleichnis“ in den Bereich der menschlichen Beziehungen zurückübertrug, in der erklärten Absicht, die Gemeinsprache um ein ,geistreiches‘ Wort zu bereichern (HA 6: 621).

Ein denkwürdiges Beispiel dafür, wie ein ,Trugbild“ (Einstein \& Infeld 1956: 149) die Forschung voranbringt und behindert, ist die von Georg Ernst Stahl (1660-1734) entwickelte Phlogistontheorie. Wörter können als ,Idole‘ oder ,falsche Begriffe، (Francis Bacon, Neues Organon) die ihnen widersprechenden Befunde überleben. Stahl erklärt die Brennbarkeit aller Substanzen durch einen in ihnen enthaltenen Stoff, das ,Phlogiston', das beim Verbrennungsvorgang entweichen soll; das Konstrukt ,Phlogiston“ beherrscht hundert Jahre lang die Vorstellungen. Experimente führen zu Hilfskonstruktionen im Rahmen dieser Theorie, der spätere Stickstoff erscheint als ,phlogistonisierte Luft', der Sauerstoff als ,dephlogistonisierte Luft‘. Aus den Aporien entwickeln Lavoisier (1743-94) und andere die Oxydationstheorie als Erklärung der Verbrennungsvorgänge und das Paradigma einer quantitativ exakt analysierenden modernen Chemie. Gleichzeitig arbeitete er mit anderen ein logisches System der chemischen Nomenklatur aus, das insbesondere durch sein Hauptwerk Traité élémentaire de chimie (1789) Verbreitung fand. Er führte die lateinisch-griechischen Komposita als Namen für chemische Verbindungen ein, ebenso das System der Vor- und Nachsilben. Außerdem vereinheitlichte er die Benennung der zu seiner Zeit bekannten Elemente. Berzelius führte 1814 die Abkürzungssymbole, Formeln und Gleichungen als die Zeichensprache der Chemie ein. Auch in diesem Fall wird ein neues Erfahrungsmodell und eine treffsichere Begriffs- und Beschreibungssprache zum Anstoß und Instrument einer enormen Wissenserweiterung, zieht eine Flut von Entdeckungen nach sich und geht auf dem Weg der Übersetzungen und Popularisierungen in die deutsche Bildungs- und Gebrauchssprache über.

Die Chemie bietet das Musterbeispiel einer internationalen, selbsterklärenden, durchsichtigen Nomenklatur. Ihr Wortmaterial ist überwiegend lateinisch/ 
griechisch. Sie arbeitet vorwiegend mit durchsichtigen Komposita, die den Schluss auf die Bestandteile einer Zusammensetzung erlauben (Natriumchlorid z. B. besteht aus Natrium und Chlor), mit Prä-, In- und Suffixen (sie sagen etwas aus über das Verhältnis, in dem die Elemente zusammengesetzt sind), mit Abkürzungssymbolen (den Anfangsbuchstaben der lateinischen Elementnamen), mit ,Formeln', d.h. Zusammensetzungen aus diesen Symbolen, die zugleich mit einem Zahlenindex versehen sind wie $\mathrm{CO}_{2}, \mathrm{NH}_{3}$ (aus ihnen lassen sich nicht nur die Bestandteile einer chemischen Verbindung entnehmen, sondern auch die quantitative Zusammensetzung ihrer Moleküle), mit ,Gleichungen“ (die chemischen Reaktionen lassen sich mit Hilfe des Plus- und Gleichheitszeichens beschreiben: z. B. $\mathrm{C}+\mathrm{O}_{2}=\mathrm{CO}_{2}$ ), mit ,Strukturformeln', die den Bau eines Moleküls graphisch als Zusammensetzung von Bausteinen darstellen, und mit dreidimensionalen räumlichen Modellen, die ihn abbilden. Diese Nomenklatur erlaubt, selbst die kompliziertesten Verbindungen in einem kurzen Ausdruck oder einer knappen Formel durchsichtig darzustellen.

In der Folgezeit wird die Fachsprache der Chemie zunehmend ein Mischtyp, z. B. als Physikalische Chemie, Biochemie, Geochemie (vgl. Fluck 1976: 83ff.). Der Umfang ihres Wortschatzes - er wurde 1964 auf 2 Millionen geschätzt (vgl. Bach 1965: 411) - und sein Anteil an der Bildungssprache oder allgemeinen Gebrauchssprache ist, aufgrund ihrer weiten Anwendungsbereiche, vermutlich der größte (vgl. Crosland 1962; Wolff 1971).

\section{0 Übertragungen von Terminologien und Denkmodellen in Nachbarfächer und in die Gemeinsprache. - Darwin, Freud, Sprachwissenschaft}

Seit dem Aufstieg der Naturwissenschaften im 17., 18., 19. Jahrhundert gibt es hinsichtlich der Darstellungsform zwei gegenläufige Bewegungsrichtungen. Einerseits beginnt seit den Akademiegründungen im 17. Jh. (vgl. 2.1) die Lösung der Naturwissenschaften von Theologie, Rhetorik, Poesie, die Verbindung von Erkenntnisanspruch und Orientierungsverzicht, der Reputationsverlust des schriftstellernden Naturwissenschaftlers (Lepenies 1976: passim). Andererseits birgt der im Namen der Aufklärung vollzogene Übergang vom Gelehrtenlatein in die Landessprachen eine Dynamik, die sich in umgekehrter Richtung auswirkt. Die Naturwissenschaften erreichen im 18. Jahrhundert zunehmend das allgemeine Lesepublikum der Gebildeten. Damit wandelt sich die Rolle des Naturwissen- 
schaftlers; im Zuge der Säkularisierung übernimmt er zunehmend Aufgaben, oder lässt sie sich aufdrängen, die vorher von anderen Instanzen versehen worden waren: die Funktion der allgemeinen Weltinterpretation. Mit dem Aufstieg der Naturwissenschaften beginnt auch die Zeit des naturwissenschaftlichen Schriftstellers. Dieser Rollenwandel wirkt zurück auf den Stil der Autoren selbst. Er bringt die zu Recht bewunderte Fachprosa des 19. Jahrhunderts hervor, aber auch eine öffentlichkeitswirksame Ungenauigkeit des naturwissenschaftlichen Schriftstellers, die erlaubt, seine Begriffsprägungen und Denkmodelle über die Grenzen des in ihnen abgedeckten Fachgebiets hinaus in andere Gebiete zu übertragen. Gemeinsprachliche Termini haben, wie erwähnt, eine erhöhte Chance verzerrter Rezeption und Wirkung.

4.1 Die Schlüsselbegriffe der Evolutionslehre Darwins sind metaphorische Termini: ,Struggle for Life“ und ,Natural Selection“ sind dem Bereich menschlicher Beziehungen und Tätigkeiten entlehnt. Der Begriff ,Natural Selection‘ wird von Darwin in Analogie zu dem menschlichen Züchter gebildet. Der Begriff ,Struggle for Life', der die treibende Kraft in diesem Auslesevorgang bezeichnet, entstand unter dem Eindruck der Bevölkerungslehre von Malthus. Im Gegensatz zu dieser pessimistischen Bevölkerungslehre sah Darwin allerdings den Konkurrenzdruck in der Natur als Vehikel des Fortschritts. Die Hauptbegriffe Darwins sind also anthropomorphe bzw. (Topitsch) ,soziomorphe‘ Denkmodelle; ihr metaphorischer und deshalb nur teilweise adäquater Charakter wird von Darwin betont. Der Begriff ,Struggle for Life' hebt an einem komplexen und umfangreichen Vorstellungsbezirk ein gemeinsames Merkmal hervor, etikettiert diesen Vorstellungsbezirk in zuspitzender Weise und umfasst ihn so in einem Gesamtüberblick. Verbindet sich diese ,pointierende Abstraktion' mit einem unscharfen Bild- und einem starken Gefühlsgehalt, so gerät er in die Nähe dessen, was man als Definition des Schlagworts bezeichnen kann. Diese Tendenz zur abgelösten, zugespitzten Formel wird verschärft durch die Übersetzung. Darwin gebraucht in der Regel ,Struggle for Life“ und unterscheidet davon ,fight‘ oder ,war'. Der erste Übersetzer, Bronn, gebraucht nebeneinander ,Ringen ums Dasein“ und ,Kampf ums Dasein', d.h. in der Übersetzung wird die Bedeutung verschoben in Richtung ,feindliche Auseinandersetzung‘. Eine semantische Verschiebung erfährt auch der Terminus ,Natural Selection“, der zunächst durch ,Natürliche Zuchtwahl' und ,Natürliche Auslese ‘ wiedergegeben wird und dann als ,Natürliche Zuchtwahl` fest wird. Der passivische Sinn von ,Auslese“ als Vorgang wird ersetzt durch einen eine aktiv handelnde Instanz suggerierenden Bedeutungsanteil. - Darwins Formeln wurden sehr bald von anderen Disziplinen aufgenommen, u. a. auch von Sprachwissenschaft und Astronomie; insbesondere wurde sie schlagartig in den Bereich der Sozialtheorie und Politik übertragen, in einer ausufernden ,sozialdarwinisti- 
schen' Literatur. - Dabei stellte sich ein doppeltes Missverständnis ein: 1. ,Struggle for Life، galt nun dem Überleben der Individuen und Gruppen, nicht wie bei Darwin der ,Art'; 2. sein biologisches Gesetz, das einen kausalmechanischen Prozess formuliert, wurde umfunktioniert zu einem Sozialprogramm. Bei der Entlehnung zeigt sich ein beträchtlicher Interpretationsspielraum der Metaphern und Modelle Darwins; aber ihre Eignung, in einer Epoche wirtschaftlicher, sozialer und politischer Konkurrenzkämpfe im Sinne legitimierender Ideologie instrumentalisiert zu werden, liegt auf der Hand. Beim Wiedereintritt in die Umgangssprache werden ,Natürliche Zuchtwahl' und ,Kampf ums Dasein“ zu einem positiv wert- und gefühlsbesetzten, begrifflich undeutlichen, abstrakten und allgemeinen Lebensgesetz. Die Übertragung der Begriffe aus der Biologie in den Sozialbereich war eine - nur halb bewusste - Rückübertragung. Begriffe aus dem menschlichen Alltagsbereich wurden zuerst in den biologischen transferiert, sie füllten sich hier mit einem genauen, vielfach belegten Inhalt, wurden zum formelhaften Ausdruck eines allgemeinen kausalmechanischen Gesetzes: die Vorstellung ,Fortschritt durch Kampf ums Dasein und Zuchtwahl` konnte nun zurückübertragen werden in den menschlichen Bereich, von dem her ihnen immer eine gewisse bildhafte Unschärfe und Gefühlsbesetzung angehaftet hatte, und konnte sich hier im Sinn einer biologischen Terminologisierung und Sanktionierung der alltagssprachlichen Vorstellungen auswirken. Die vorherige Projektion begünstigte eine Rückprojektion. Die Übereinstimmung in der Sprache der beiden Bereiche legte eine Kongruenz in der Sache nahe. Die anthropomorphe bzw. soziomorphe Zoologie schlug um in eine zoomorphe Anthropologie und Sozialtheorie.

Was sich bei Darwin nur andeutet, die Nahtstelle, wo Wissenschaft in allgemeine Weltanschauung übergeht, erscheint als Erklärungsmodell für etwas, was sich in der Geschichte der Biologie ständig und auf breiter Ebene wiederholt und zu zahlreichen Entlehnungsvorgängen geführt hat. Man beobachtet es an den stehenden Redewendungen der populärwissenschaftlichen und öffentlichen Diskussionen vor 1914 (,unerbittlicher Kampf ums Dasein‘, ,Auslese des Passenden, des Nützlichen, des Zweckmäßigen', ,Vervollkommnung durch Zuchtwahl‘, ,Herdentrieb“, ,Platz an der Sonne‘), an der ,Staatsbiologie‘ und Biologisierung der öffentlichen Sprache nach 1933 (,reinrassig‘, ,ausmerzen‘). Das Phänomen von Übertragung und Rückübertragung ist ebenso im Bereich der Biosoziologie und Verhaltensforschung zu beobachten (,Tierstaat', ,Termitenstaat', ,aggressives Verhalten', ,soziale Hierarchie‘, ,Demutsverhalten‘, ,Hackordnung“, ,Imponiergehabe').

4.2 Der Psychologe ist, da sein ,Gegenstand“ ungegenständlich ist, auf Metaphorik angewiesen. Freud hat seine Vergleiche sehr verschiedenen Bereichen entlehnt, wählt aber bevorzugt Analogien, die den Naturwissenschaften entnommen sind. 
Die Entlehnung erstreckt sich nicht nur auf einzelne Vokabeln, sondern öfter auf ein ganzes Wortfeld und einen Darstellungstypus. Der Terminus und das Bildfeld ,Psychoanalyse' entstammen der Chemie. Die Drei Abhandlungen zur Sexualtheorie (1905) folgen dem Sprachtypus der biologischen Systematik. Freuds Vorstellung von der Psyche als eines Apparats, in dem messbare oder jedenfalls in ihrer Quantität abschätzbare Energien umlaufen und sich verteilen, abreagiert und verschoben, erhöht und verringert werden können, die Idee von der Psyche als eines Energieverteilungsapparats ist der Physik entlehnt und hat den Wert eines einen umfangreichen Bezirk erschließenden Denkmodells. Bevorzugt Freud den naturwissenschaftlichen Sprachtyp als eine Form der Prestigeanleihe, die dazu dient, einer neuen, unerprobten Forschungsrichtung die Autorität und Legitimation eines naturwissenschaftlichen Fachs zu verleihen? Die naturwissenschaftlichen Analogien sind wohl insgesamt mehr als bloße Metaphern. - Freud verstand die Tiefenpsychologie als Teildisziplin der Naturwissenschaft und sich als Naturwissenschaftler, der nur noch nicht in der Lage sei, die psychischen Vorgänge bis in ihre physiologische, physikalisch-chemische Basis zu verfolgen. Er bevorzugt eine naturwissenschaftliche Sprache, weil er ihr den höchsten Annäherungswert an die tatsächlichen Zusammenhänge zuschreibt. Die Frage, ob ein solches Bildfeld und Denkmodell das Denkobjekt reduziert, verzeichnet, oder bedeutungslos bleibt, ist mehrfach diskutiert worden. Habermas bringt das Problem auf die Formel eines „szientistischen Selbstmißverständnisses der Psychoanalyse“ und ist der Meinung, dass die naturwissenschaftliche Sprache Freuds einen nur scheinbaren Anschluss an das naturwissenschaftliche Weltbild der Jahrhundertwende herstelle. Vermutlich begünstigte die naturwissenschaftliche Metaphorik Freuds seine Aufnahme bei den Anhängern des mechanistischen Weltbilds der Jahrhundertwende und erhöhte seine Chance, zum Weltanschauungssurrogat zu werden, während sie bei den Gegnern dieses Weltbildes von vornherein zu der Weigerung führte, den Kern der Entdeckungen Freuds zur Kenntnis zu nehmen. In drei Entlehnungsschüben, vor 1914, in den zwanziger Jahren und seit 1968, ist ein beträchtlicher Teil des Freudschen Vokabulars in die Bildungs- und Umgangssprache übergegangen. Speziell Vokabeln aus dem Bereich der Biologie, Chemie, Physik und Technik sind auf dem Umweg über Freud Bestandteil der ,Alltagspsychologie' geworden: ,Trieb', ,Aggressionstrieb', ,Todesstrieb', ,Destruktionstrieb', ,Geschlechtstrieb', ,Liebestrieb‘, ,Libido', ,Affekt', ,Psychoanalyse', ,Analytiker', ,analysieren', ,Sublimierung', ,fixiert sein', ,psychische Energie und Arbeit', ,bearbeiten', ,aufarbeiten', ,Trauerarbeit', ,Fehlleistung', ,psychische Mechanismen', ,Abwehrmechanismen', , abreagieren', ,projizieren‘.

4.3 Auch die Linguistik bedient sich in Abständen naturwissenschaftlicher Metaphern. Die ,Lautgesetze‘ der Junggrammatiker oder Tesnières Begriff der Verbva- 
lenz sind zwei Beispiele. Die Richtungen der Sprachwissenschaft, die sich Mitte der 1960er Jahre als ,moderne Linguistik' eingeführt haben, bevorzugen in weitem Maße den naturwissenschaftlichen Sprachtyp und formalisierende Schreibweisen. Soweit es sich dabei nicht um Formen der Prestigeanleihe handelt zahlreiche linguistische Veröffentlichungen verdanken ihre Existenz fast ausschließlich der Prestigeanleihe bei einer verbreiteten Vorstellung von Exaktheit stellt sich die Frage nach einem ,szientistischen Selbstmissverständnis‘ der Linguistik. Jedenfalls werden offenkundige Nachteile dieser Vernaturwissenschaftlichung erkennbar. Der Sinn einer Humanwissenschaft, die der Selbstverständigung dient, kann sich nur in einer verständlichen Sprache erfüllen, die den Gebrauch der fachsprachlichen Mittel auf das, was im Hinblick auf die Sacherschließung notwendig und im Hinblick auf den Zweck der Mitteilung sinnvoll ist, beschränkt. Die gemeinsprachliche Ausdrucksweise scheint überdies besser geeignet, die im Vergleich zu den naturwissenschaftlichen ungleich größere Komplexität unserer Gegenstände abzubilden als eine, die sich relativ einfacher Schreibweisen der Naturwissenschaft bedient und dadurch ihren Gegenstand reduziert und verfremdet und seine Darstellung verumständlicht. Nur eine verständliche Sprache ermöglicht zudem ein fachübergreifendes Gespräch und den Übergang sprachwissenschaftlicher Begriffe in die Gemeinsprache.

Bei dem Übergang von Terminologien in Nachbarfächer und in die Gemeinsprache gibt es das Phänomen von ,Schub“ und ,Sog‘, von ,Expansion“ der Ausgangssphäre und ,Attraktion“ durch die Zielsphäre. Erfolgreiche Konzepte „mit bewährter Kraft der Weltaufschließung“ (Trier) haben die Tendenz, überzugehen in Nachbarfächer und in die Gemeinsprache; ein Begriffsapparat, der sich in einem Fach bewährt hat, neigt dazu, zu wandern, von anderen Bereichen entliehen zu werden. Es ist ein Vorgang der Entlehnung - innerhalb der Schichten und Gruppen einer Sprachgemeinschaft, eine Form des ,social borrowing‘: „Bei Entlehnungen kommt es vor allem auf Prestige an“, meint Ullmann; übernommene Wörter evozieren das Prestige ihres ursprünglichen Milieus. - $\mathrm{Zu}$ dem sachaufschließenden Wert eines Terminus und seinem Prestige kommt als weitere Ursache für die Entlehnung sein sozialer Gebrauchswert. 


\subsection{Jahrhunderttypische Wortschatz- erweiterungen, Wege und Ursachen der Entlehnungen, Typen der Bedeutungs- veränderung beim Übergang in die Gemeinsprache}

Der unüberschaubare Wortschatz, der aus den Naturwissenschaften in die Gemeinsprache entlehnt worden ist, ist von dieser her kaum zu ordnen. Nur einige Gesichtspunkte, unter denen eine Gliederung möglich scheint, seien angedeutet.

5.1 In dem Wandel des Wortschatzes, in der Ersetzung z.B. des mittelalterlichen ,Meergarten' (ahd. ,merigarto‘, mhd. ,mergarte'), der meerumschlossenen Erdscheibe, durch den neuzeitlichen ,Erdball‘, spiegelt sich der Wandel der Epoche. In der Erweiterung des deutschen Wortschatzes wird die Erweiterung des theoretischen und konkreten Horizonts erkennbar. Charakteristisch für das 15. und 16. Jahrhundert ist z.B. die Entlehnung mathematischer Begriffe aus dem Lateinischen, in der Form der Assimilation (,multiplizieren', ,addieren‘, ,dividieren') oder der Lehnübersetzung (,Nenner‘, ,Zähler‘, ,Viereck‘, ,Dreieck‘ aus lat. ,denominator', ,numerator', ,quadrungulus‘, ,triangulus'), die umfangreiche Wortschatzerweiterung im Grenzbereich von Alchemie und Heilkunst durch Paracelsus zu Beginn des 16. Jh.s (vgl. 2.2), und die in dem geographischen Ausgriff begründete Bereicherung um Konkreta und Abstrakta: ,Ananas‘ (bras.), ,Bambus‘ (ndl.), ,Banane‘ (port./span.), ,Kanarienvogel` (span.), ,Lama‘ (span., aus dem Peruanischen), ,Äquator‘ (lat.) und ,Geographie‘, ,Horizont‘, ,Klima‘, ,Zenit‘ (jeweils griech.). - Ebenso sprechend sind Wortschatzerweiterungen in den folgenden Jahrhunderten. Aus dem 17. Jahrhundert nenne ich: ,Axiom‘, ,Barometer', ,Baumschule‘, ,Botanik‘, ,Experiment‘, ,Fernglas‘, ,Fernrohr‘, ,legieren‘, ,Mikroskop‘, ,Nikotin‘, ,Optik‘, ,Skala‘, ,Skelett‘, ,Schlüsselbein‘. Für das 18. Jahrhundert mögen folgende Wortgruppen stehen: ,Amphibie‘, ,botanisieren‘, ,Fauna‘, ,Instinkt‘, ,Organ‘, ,Parasit‘, ,Zelle‘, ,Zoologie‘; ,Bauplan der Wirbeltiere‘ und ,Metamorphose der Pflanzen` (Goethe); ,Blitzableiter‘ und ,Dampfmaschine‘ (Lehnübersetzung von engl. ,steam-engine‘, Lichtenberg); ,Brechung‘, ,Lichtstrahl‘, ,Spektrum‘, ,Schwerkraft‘, ,Zentrifugalkraft‘; ,Sauerstoff‘, ,Wasserstoff‘ (Lehnübersetzungen aus frz. ,oxygéne‘, ,hydrogene‘), ,Stickstoff‘ (Lehnübersetzung nach frz./engl. ,azote` = Stoff, in dem kein Leben möglich ist). - Im 19. Jahrhundert wächst die Zahl der nennbaren Fachausdrücke sprunghaft; z.B.: ,Aluminium‘, ,Atomistische Hypothese‘, ,Bazillus‘, ,Dampfschiff‘, ,Dynamik‘, ,Dynamo‘, ,Elektrotechnik‘, ,Elementaranalyse‘, ,Energieprinzip‘, ,Evolutionstheorie‘, ,Helium‘, 
„Homöopathie‘, ,immun', ,Jod‘, „Kali‘, ,Kraftfeld‘, ,Nitroglyzerin‘, ,Plankton“, ,Röntgenröhre‘, ,Spektralanalyse‘, ,Telegraph‘. - Aus der Wortschatzerweiterung des 20. Jahrhunderts seien nur wenige Beispiele genannt: ,Antenne‘, ,Atomkraft‘, ,Gammastrahlen‘, ,Genetische Information‘, ,Glühbirne‘, ,H-Bombe‘, ,Kernenergie‘, „Kernfusion‘, ,Kernspaltung‘, ,Laserstrahlen‘, ,Ökologie‘, ,Quantentheorie‘, ,Transistor‘, Ultraschall‘, ,Uranspaltung‘, ,Verhaltensforschung‘ (vgl. 5.2).

5.2 Als die Hauptursache der Entlehnungen erscheint ihr sozialer Gebrauchswert, ein „Sog“ also, der von den verschiedensten Anwendungsbereichen ausgeht. Verwertbare Erfindungen, Entdeckungen und grundsätzliche Veränderungen des Naturbildes werden zum gebrauchten und bekannten Sachgut und Bildungsgut. Eine vollständige Erfassung der Übergangswege würde eine systematische Auffächerung unserer ganzen Lebenswelt bedeuten. Jacob Burckhardts (Weltgeschichtliche Betrachtungen, 2. Kap.) Unterscheidung der „drei Potenzen“ - Religion, Staat, Kultur - ist hilfreich.

a) Ein Motiv für die Verbreitung eines naturwissenschaftlichen Weltbilds ist das „metaphysische Bedürfnis“. Statt bei Religion und Philosophie sucht man Orientierung und Wahrheit bei den Naturwissenschaften, sei es im Zuge optimistischen Fortschritts- und Erkenntnisglaubens gegen Ende des 19. Jahrhunderts (Haeckels Welträtsel, Büchners Kraft und Stoff), sei es auf der Grundlage eines pessimistischen Krisengefühls gegen Ende des 20. Jahrhunderts (Lorenz, Die Rückseite des Spiegels).

b) Eine zweite Übergangsstelle ist der Staat. Der Staat sucht naturwissenschaftliche Erkenntnisse und deren Ansehen zu instrumentalisieren und durchsetzt die offizielle Sprache ,naturwissenschaftlich“ (extrem im NS-Staat, vgl. 4.1), oder er betreibt Politik aufgrund wissenschaftlicher Beratung und unterstützt die staatlich effektive Naturwissenschaft. Über seine Ressorts, ,Verteidigung‘ oder ,Wissenschaft und Technologie،, ,Gesundheit‘ oder ,Landwirtschaft und Ernährung،, dringt naturwissenschaftlich-technisches Vokabular ins allgemeine Bewusstsein, von der ,Neutronenbombe ' und der ,Glasfiber' der neuesten Nachrichtentechnik bis zum ,Schweren Wasser‘ und ,Atommüll‘, zur ,ökologischen Katastrophe‘ und zur ,Arsenschlämme‘, die die Trinkwasserversorgung gefährdet. -

c) Die Kultur leistet, in unterschiedlicher ,Tiefenschärfe‘ (Habermas 1978: 330), die breiteste Orientierung. In diesem heterogenen Bereich gibt es zahlreiche Vermittlungsinstanzen. Das staatliche Unterrichtswesen erreicht seit Ende des 19. Jahrhunderts, dass auch speziellere Vokabeln wie ,Plankton', ,Selektion“, ,Mutation‘, ,Chromosomen“, ,Doppel-Helix‘, ,Atommodell‘ als wissenschaftliche Begriffe vermittelt werden und in einen engeren Bildungswortschatz eingehen. Eine erhebliche Rolle bei der Vermittlung naturwissenschaftlicher Allgemeinbildung spielt auch die Bildungsindustrie, der Bestandteil naturwissenschaftlicher 
Information in Fernsehen, Rundfunk, Zeitung, Zeitschrift, Sachliteratur (vgl. 6.). Auf dem Weg der aktuellen und sensationellen Information dringen indirekt Vokabeln wie ,Raumsonde‘, ,Hochdruckgebiet‘ und ,Meteorologie‘, ,Arsen“, ,Blausäure‘, ,Zyankali‘, ,Nitroglyzerin‘ und ,Dynamit‘, ,LSD“ und ,Cocain‘, ,Plasmaphysik‘ oder ,Strontium‘ kurz- oder langfristig ins allgemeine Bewusstsein. Die Entwicklung der Wirtschaft und Technik und ihre Absatzwerbung, der ökonomische Gebrauchswert der Naturwissenschaften, bewirkt die quantitativ stärkste Verbreitung naturwissenschaftlichen Vokabulars, sei es im Bereich der Optik (,Belichtungsmesser‘, ,Teleobjektiv') oder des elektrischen Netzes (,Volt‘, ,Ampere‘, ,Schalter‘, ,Transformator'), der Landwirtschaft (,Nitratdünger‘, ,Phosphatdünger‘, ,Stickstoffdünger‘, ,Herbizide‘) oder der Arzneimittelindustrie (,Chinin“, ,Serum‘, ,Aspirin`). Die ärztliche Versorgung hat dazu geführt, dass ein heutiger Sprachbenutzer über seine biologischen Körperfunktionen (,Darmflora‘, ,Herzkranzgefäße‘, ,Vegetatives Nervensystem‘, ,Viren') häufig in wissenschaftlichen Termini orientiert ist. Die Verwissenschaftlichung reicht von der Religion und Politik über das Bildungswesen, die Bildungsindustrie und die Wirtschaftswerbung bis in die Berufspraxis. Einer Verwissenschaftlichung des Alltags entspricht eine Verwissenschaftlichung der Umgangssprache und ein erhebliches Gewicht eines halb, nur in seiner sozial relevanten Komponente verstandenen und passiven Wortschatzes.

5.3 Den Übergangswegen der Popularisierung entsprechen zumindest tendenziell unterschiedliche Texttypen:

a) Der Typus naturwissenschaftlicher Darstellung als Weltanschauungssurrogat, meist als Kontrafaktur zur Bibelsprache;

b) die von naturwissenschaftlichem Vokabular durchsetzte politische Rede oder Programmschrift;

c) das Schulbuch und Lehrbuch, die Sachliteratur (vom Fachlexikon über das ,erzählerische Sachbuch` bis zum Bildsachbuch), die Sendung aus dem Bereich der Naturwissenschaft in Funk, Fernsehen, Film, der Wissenschaftsbericht in Zeitungen und populären Zeitschriften, der aktuelle Bericht über Entdeckungen oder Katastrophen im Anwendungsbereich der Naturwissenschaften, die Wettervorhersage, die von naturwissenschaftlichem Vokabular und den Formen naturwissenschaftlicher Wortbildung durchsetzte Werbung, die für die Berufspraxis bestimmte Fachliteratur. Auch die schöne Literatur, der Roman z. B. als Vermittler naturwissenschaftlicher Inhalte ist hier zu nennen.

Es wäre eine eigene Aufgabe, die an den Texttypen zu beobachtende Stildifferenzierung zu betrachten: das Verhältnis von Information und Redundanz, die übersichtliche oder verstreute Anordnung der Information, das Niveau der sachlichen und sprachlichen Voraussetzungen und die Kompliziertheit der Darstellung, 
den Anteil der optischen Vermittlungen von Information und die Art der Verbildlichung. Syntax, Idiomatik und Metaphorik unterscheiden sich nach der Intention der Texte und der Breite des intendierten Publikums. Die Dichte des fachspezifischen Vokabulars und die Art seiner Verwendung steht im Zusammenhang mit dem jeweiligen Texttyp und seiner Popularitätsstufe.

5.4 Beim Übergang eines Fachausdrucks aus der Naturwissenschaftssprache in die Bildungs- und Umgangssprache kann dessen Bedeutung konstant bleiben. Das gilt offenbar mehr für den Bereich der Gegenstandsnamen (,Pyrit‘, ,Bunsenbrenner', ,Barometer', ,Jod') als für den der Begriffsbezeichnungen. In der Regel führt der Sphärenwechsel anscheinend zu einer gewissen Bedeutungsveränderung. Statt des Sachbezugs werden stärker die in dem Sozialrahmen der Bildungsund Umgangssprache erheblichen Bedeutungsaspekte aktualisiert. Man kann einige (im Einzelfall oft kombinierte) Typen von Bedeutungsverschiebung unterscheiden: wenn ein Begriff der Alltagssprache terminologisiert wird, erfährt er eine Spezialisierung der Bedeutung durch Verengung des Umfangs und Bereicherung des Inhalts. Der Assoziationshof von Gefühlen und Wertungen, das Konnotat, wird so weit wie möglich ausgeblendet (z. B. ,Widerstand' in der Elektrizitätslehre; ,Art‘, ,Bastard‘, ,Kultur‘, ,ökologische Nische‘, ,Reizbarkeit‘, ,Trieb“ in der Biologie).

Wenn ein Begriff der Wissenschaftssprache in die Populärwissenschaft und in die Umgangssprache übergeht, geschieht oft das Umgekehrte: $a$. Der Begriff erfährt eine Bedeutungserweiterung, eine Erweiterung des Umfangs und eine Verarmung des Inhalts. Er wird breit und vielseitig verwendet und verliert den spezifischen, klaren begrifflichen Inhalt (z.B. beim Übergang der alchemistischen Begriffe ,Elixier‘ und ,Essenz‘, der biologischen Begriffe ,Art‘ und ,Trieb“, der psychoanalytischen Begriffe ,Widerstand' und ,Trauma' in die Umgangssprache). - $b$. In der aus der Naturwissenschaft entlehnten Metapher - die Grenze zum metaphorischen Gebrauch ist fließend - wird die Bedeutung ebenfalls oft erweitert (,Machtvakuum‘, ,Keine Experimente!‘, ,Sublimierung“, ,menschliches Klima und gute Atmosphäre‘, ,enges Spektrum‘). - c. Der Begriff behält u. U. seinen Anwendungsbereich und wird kontextadäquat eingesetzt, sein Inhalt erscheint aber als nebuloser oder weißer Fleck und kann im Zweifelsfall nicht definiert werden. Der Umgang mit ,Pseudobegriffen‘ (,Kernreaktor‘, ,Alchemie‘, ,Relativitätstheorie') ist überaus häufig. - $d$. Der Begriff wird von den Wortbestandteilen her interpretiert, also etymologisch aufgefasst. Bei Bekanntschaft mit der Sache definiert sich der Inhalt eines Begriffs von den Sachzusammenhängen her. Die motivierte Bildung wird dann nicht mehr aus den Bedeutungen ihrer Bestandteile erschlossen, sie verliert ihre Durchsichtigkeit zugunsten der in ihr intendierten Sache. Diese Sachsteuerung des Verstehens funktioniert aber nur, solange und 
soweit die Sache bekannt ist, und auch dann nur halb. Je weniger die Sache bekannt ist, um so mehr gewinnt ein wortgesteuertes Verstehen die Oberhand. Es wird besonders durch metaphorische Termini begünstigt. Sie knüpfen an bekannte Vorstellungen an und enthalten damit einen Hinweis auf die gemeinten begrifflichen Inhalte, man versteht sie halbwegs und wird sehr leicht zu der vorschnellen Meinung verführt, sie ganz zu verstehen, die Sache in dem Wort zu haben (vgl. die Wirkung der Begriffe ,Kampf ums Dasein‘, ,Atomkern‘, ,Kernspaltung‘). - e. Die Begriffe nehmen umgangssprachlich einen Assoziationshof von Gefühlen und Wertungen an, die dem neuen Sozialfeld angehören. Sie werden konnotathaltig sowohl im aufwertenden Sinn (,Dynamik‘, ,Rasse‘, ,Regeneration‘, ,Atmosphäre‘) wie im abwertenden Sinn (,Nesthocker‘, ,Domestikation', ,steril', ,Atomstaat'). $-f$. Die Begriffe transportieren das Prestige der Wissenschaftlichkeit in die Umgangssprache (,Relativitätstheorie‘, ,Astrophysik‘, ,Ethologie‘). U.U. ist das Prestige der dominierende Bedeutungsaspekt. - g. Ein charakteristisches Mittel beim Übergang in das umgangssprachliche Sozialfeld ist schließlich der Euphemismus, das beschwichtigende Hüllwort, meist in Form der positiv besetzten Metapher (,Atommeiler‘, ,Schneller Brüter‘, ,Entsorgungspark'). - Art und Grad der Bedeutungsverschiebung kann je nach Texttyp, Intention, Popularitätsgrad variieren.

\subsection{Die Entfernung der Fachsprachen von der Gemeinsprache und das Problem ihrer Übersetzung}

Seit dem Übergang aus der lateinischen in eine deutsche Naturwissenschaftssprache gibt es einen zweiten bewussten Übersetzungsvorgang, der von dem gleichen aufklärerischen Impuls getragen ist: die Übertragung des Wissenswerten aus den deutschen sich von der Gemeinsprache entfernenden Fachsprachen in eine auf ein allgemeines Lesepublikum berechnete populäre Prosa. Dieser zweite Übersetzungsvorgang ist im 20. Jahrhundert um so wichtiger geworden, als die Kluft zwischen Naturwissenschaftssprache und Gemeinsprache sich rapide erweitert hat.

6.1 Der Vorgang hat vier Aspekte: $a$. Seit der Verselbständigung der naturwissenschaftlichen Disziplinen in der frühen Neuzeit und ihrer universitären Institutionalisierung im 19. Jahrhundert haben sie sich erweitert, aufgefächert und in zahlreiche Teildisziplinen desintegriert. Zur Physik gehören z.B. Gebiete wie Molekülphysik, Theorie der Elementarteilchen, Theorie der kondensierten Materie, Astronomie und Astrophysik, zur Chemie Anorganische und Analytische 
Chemie, Organische Chemie, Physikalische Chemie, Mineralogie, Kristallographie, Biochemie, Quantenchemie, zur Biologie Allgemeine Genetik, Systematische Botanik und Pflanzengeographie, Pflanzenphysiologie und Zellbiologie, Biochemie und Molekularbiologie, Allgemeine Zoologie, Tierphysiologie und Angewandte Zoologie, Angewandte Genetik - mit jeweils eigenen Instituten und Lehrstuhlvertretern. Die Differenzierung der Fachgebiete ist begleitet von einer explosionsartigen Ausweitung des Wortschatzes der Fachsprachen. Die Wörter der Gemeinsprache unterliegen einer zunehmenden fachinternen Bedeutungsspezialisierung und die Nomenklaturen wachsen ins Unübersehbare. Die Wortschätze der Naturwissenschaften werden nach hunderttausenden bzw. nach Millionen gezählt. - $b$. Der Fachstil hat sich gewandelt. Das Prinzip der Ökonomie und Präzision führt in den Fachtexten zu einer oft schon in den Nachbarbereichen nicht mehr zugänglichen spezialsprachlichen Informationsverdichtung. Charakteristisch ist die hohe Gebrauchsfrequenz eines fachspezifischen Vokabulars und nichtsprachlicher Darstellungsmittel (Symbole, Tabellen, Diagramme), die Tendenz zur Substantivierung und zum grammatikalisierten Verb, zu informationsverdichtenden, satzabkürzenden Formen wie Passivkonstruktionen, Partizipien, Substantivgruppen, mehrgliedrigen Komposita, zur Multiplikation definierter Wortstämme durch die Mittel der Wortbildung, zu einer begrenzten Wortliste, stereotypen Satzbauplänen und einer Darstellungsidiomatik. - c. In der modernen Physik taucht seit der Formulierung der Relativitäts- und Quantentheorie das besondere Problem auf, dass das theoretisch und experimentell Erschlossene sich der Versprachlichung zu entziehen scheint, weil es mit unseren Wahrnehmungskonventionen und unserer herkömmlichen Logik nicht $\mathrm{zu}$ vereinbaren ist, die Verlegenheit, dass z. B. auch die Metaphern - der ,gekrümmte Raum‘ Einsteins eigentlich keine Vorstellung vermitteln, weil sie an Unbekanntes anknüpfen, oder dass der Physiker „zur Beschreibung der kleinsten Teile der Materie abwechselnd verschiedene, einander widersprechende anschauliche Bilder verwendet“, um sich, wo er präzis werden will, in die mathematische Kunstsprache zurückzuziehen (Heisenberg 1967b: 33f.). Der Übergang in die Nichtsprachlichkeit, in die mathematische Formulierung, gilt für den mikroskopischen Bereich auch der Biologie und Chemie, aber nicht nur hier. Der „sprachlose Gelehrte“ (Winau 1980: 330) ist in weiten Bereichen der Naturwissenschaften und Medizin im Vordringen. - $d$. Seit dem 2. Weltkrieg ist das Amerikanische/Englische zur wissenschaftlichen Weltsprache geworden; die wesentlichen Veröffentlichungen bundesrepublikanischer Naturwissenschaftler erscheinen auf englisch. Die einstige Kluft zwischen der lateinischen Gelehrtenzunft und den Laien ist in vieler Hinsicht wiederhergestellt und vertieft, das öffentliche Bewusstsein ist weit hinter den Erkenntnissen der modernen Naturwissenschaft zurückgeblieben. 
6.2 Der zweite bewusste Übersetzungsprozess, der auf diese Situation antwortet und in dem breiten Popularisierungsvorgang (vgl. 5.2, 5.3) eine Sonderstellung einnimmt, ist in den letzten Jahrzehnten intensiviert und erweitert worden. Die Sprachwissenschaft ist an diesem Prozess nicht beteiligt, und es ist auch die Frage, ob ein interdisziplinäres deutsches Wörterbuch, von Harald Weinrich 1975 vorgeschlagen und seither diskutiert (vgl. Henne 1978), angesichts der Geschwindigkeit, mit der sich gegenwärtig das lexikonwürdige Wissen erweitert - Verlage von Enzyklopädien rechnen mit einer Verdoppelung in einem Zyklus von 4-5 Jahren - die Chance hätte, $\mathrm{zu}$ einem Zeitpunkt $\mathrm{zu}$ erscheinen, an dem es nicht schon veraltet wäre. Die Übersetzung wird getragen von einer kleinen Gruppe (meist im Alter) gemeinverständlich schreibender Naturwissenschaftler, vom Hochschul- und Schulunterricht und den Verfassern von Schulbüchern und Lehrbüchern, von den nicht allzu dicht gesäten Wissenschaftsjournalisten und den Verlegern der sprunghaft angestiegenen Sachliteratur. An den verschiedenen Typen des ,Sachbuchs‘, vom Lexikon über das ,erzählerische Sachbuch“ (Hoimar von Ditfurth) zum Bildsachbuch der Mail-Order-Konzerne, lassen sich durch Vergleich von Texten, in denen dasselbe Thema wissenschaftlich und populär behandelt wird, die Übersetzungsmittel, Popularisierungsstufen, die Leistung und das Problem des Übersetzens i.e. beobachten. Die Übertragung wissenschaftlicher Prosa in ein populärwissenschaftliches Sachbuch besteht nur z. T. in einem ,Übersetzen'; in der Hauptsache ist es ein Vorgang der Umarbeitung aus einem überwiegend der Sacherschließung dienenden Darstellungstyp in einen publikumsorientierten. Ludwik Fleck (1980: 148f.), der in der populären Wissenschaft einen allgemeinwirkenden Faktor jedes Erkennens sieht, charakterisiert sie als „vereinfachte, anschauliche und apodiktische Wissenschaft“. In ihr kehren Rhetorik und Poesie zurück, von denen sich die Naturwissenschaftssprache im Zeitalter des Humanismus schrittweise emanzipiert hatte (vgl. 2.). Der Vorzug ihrer der Differenzierung unserer Sprache entgegenwirkenden Anstrengung liegt darin, dass sie fachsprachlich vorformulierte Inhalte von der Gemeinsprache her neu durchdenkt und sie allgemeiner zugänglich macht, dass sie die von der Aufklärung geforderte Teilhabe aller an dem von den Wissenschaften Erarbeiteten, die Zirkulation der geistigen Güter ermöglicht. Eine differenzierte Ausarbeitung der Gemeinsprache und ihre Bereicherung um neue Begriffe ist die Folge. Als Nachteil erscheint, je nach Marktorientierung und Popularitätsstufe, ein Zug zur Entsachlichung. Die wissenschaftliche Mitteilung verschwindet oder wird entstellt unter ihrer literarischen und ideologischen ,Verpackung‘. Der Übersetzungs- und Popularisierungsvorgang (vgl. 5.2, 5.3) insgesamt ist gleichbedeutend mit einer Verwissenschaftlichung des Alltags. Er führt zu einer Verwissenschaftlichung und streckenweise $\mathrm{zu}$ einer terminologischen Überlastung der Umgangssprache. 
Leibniz entdeckte die Sachprosa als Mittel der Sprachbildung und forderte einen Kanon vorbildlicher ,Kernschriften'. Tatsächlich ist der Kanon, der für unsere Einheitssprache normbildend wirkte, dann ganz überwiegend ein literarischer gewesen: er bestand aus den Werken unserer Klassik. Deren Sprache weist, von der Gegenwart her gesehen, bei aller Vorbildhaftigkeit große Lücken auf - es fehlen weite Sachbereiche, wie z.B. die der Naturwissenschaften - und diese Lücken sind wohl auch kaum zu schließen durch die Belletristik der Gegenwart. Es wäre förderlich, den Gedanken der sprachbildenden Wirkung der Sachprosa mehr als bisher zu beherzigen und die vorbildliche naturwissenschaftliche Prosa, von Newton bis zu den Autoren des 20. Jahrhunderts, als Literatur zu würdigen. 